RICE UNIVERSITY

\title{
Scheduling with QoS in Parallel I/O Systems
}

by

\author{
Ajay Gulati
}

A Thesis Submitted

IN PARTIAL FULFILLMENT OF THE

REQUIREMENTS FOR THE DEGREE

Master of Science

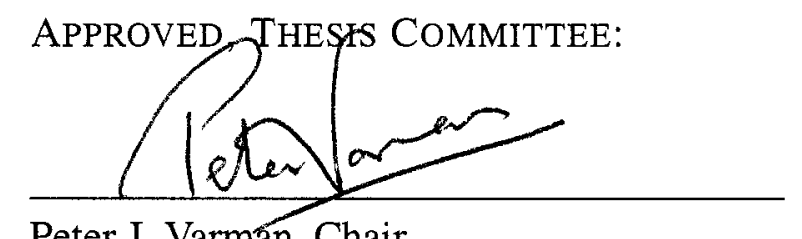

Peter J. Varman, Chair

Associate Professor of Electrical and

Computer Engineering and Computer

Science

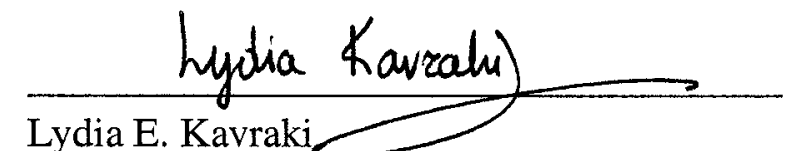

Professor of Computer Science

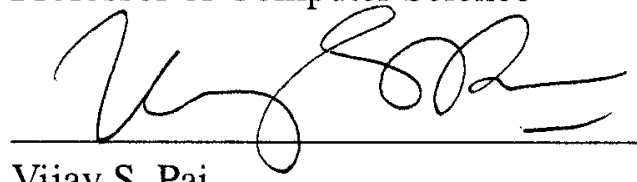

Vijay S. Pai

Assistant Professor of Electrical and

Computer Engineering and Computer

Science

Houston, Texas

July, 2004 
UMI Number: 1425836

\section{INFORMATION TO USERS}

The quality of this reproduction is dependent upon the quality of the copy submitted. Broken or indistinct print, colored or poor quality illustrations and photographs, print bleed-through, substandard margins, and improper alignment can adversely affect reproduction.

In the unlikely event that the author did not send a complete manuscript and there are missing pages, these will be noted. Also, if unauthorized copyright material had to be removed, a note will indicate the deletion.

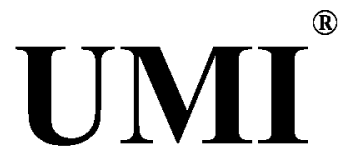

UMI Microform 1425836

Copyright 2005 by ProQuest Information and Learning Company. All rights reserved. This microform edition is protected against unauthorized copying under Title 17, United States Code.

ProQuest Information and Learning Company 300 North Zeeb Road

P.O. Box 1346

Ann Arbor, MI 48106-1346 


\section{Scheduling With QoS in Parallel I/O SYSTEMS}

Ajay Gulati

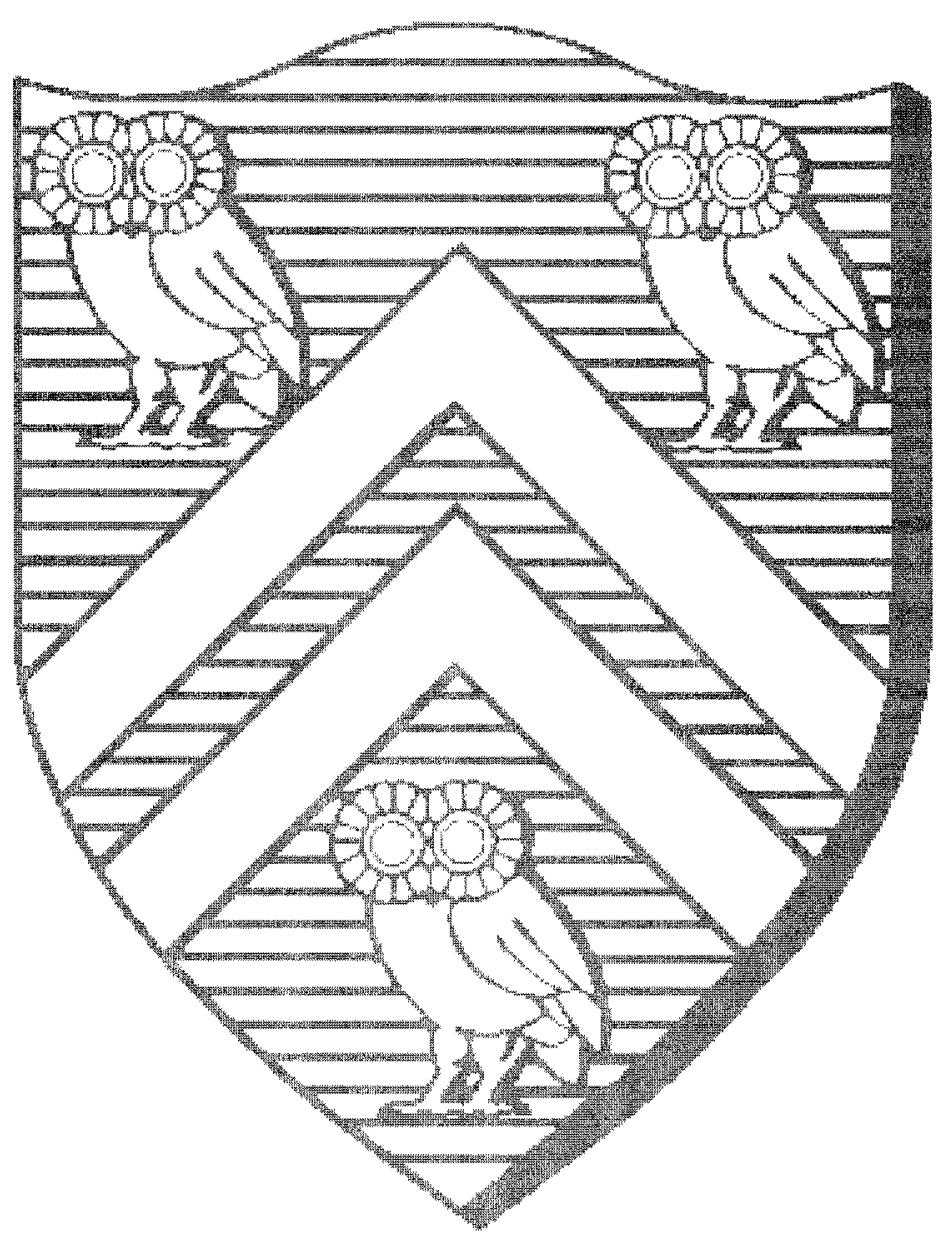

Thesis: Master of Science

Computer Science

Rice University, Houston, Texas (December 2004) 


\title{
Scheduling with QoS in Parallel I/O Systems
}

\author{
Ajay Gulati
}

\begin{abstract}
Parallel I/O architectures have become attractive in the context of high performance computing and services provided by high bandwidth data centers. It is challenging to provide a scheduling technique that maximizes throughput and can provide QoS guarantees. In this thesis we first introduce the problem of maximizing the throughput for a parallel $\mathrm{L} / \mathrm{O}$ system that is simultaneously accessed by several concurrent applications. We show that the problem of obtaining a minimum length schedule is NP-complete. We present faimess metrics for parallel $\mathrm{I} / \mathrm{O}$ and provide fair scheduling schemes for some representative situations. In particular we study schemes that support fairness at every I/O step (local fairness) and during a certain window of time (global fairness). We also present a more general algorithm for weighted allocation of disk system bandwidth to multiple reference strings. All the three algorithms have low polynomial time complexity and they are work conserving.
\end{abstract}




\section{Acknowledgments}

First and foremost, I thank my advisor Professor Peter Varman for his guidance and the numerous discussions we had about all the algorithms presented in the thesis. I am also thankful to him for all the travel he did to be able to have regular meetings. His support and insightful views helped me a lot in shaping my research direction.

I am grateful to Sumit Mittal and Anwis Das for their useful comments on the initial draft of the thesis. I am also grateful to my colleagues Animesh, Atul, Johnny, Cristi, and Yuri for the useful discussions I had with them.

Last but not least, I also thank Professor Lydia Kavraki and Professor Vijay Pai for agreeing to be in my thesis committee and giving me useful reviews and suggestions, which greatly improved the quality of this thesis, despite their heavy schedules.

Research support from the National Science Foundation is gratefully acknowledged. 


\section{Contents}

Abstract $\quad$ ii

Acknowledgments

List of Illustrations $\quad$ vi

1 Introduction 1

1.1 Overview of the Thesis .................. 4

1.2 Major Contributions . . . . . . . . . . . . . . 5

1.3 Previous Work . . . . . . . . . . . . . . . 7

1.3.1 Parallel Disk Scheduling . . . . . . . . . . . . . 7

1.3.2 QoS Scheduling ..................... 9

2 Models and Definitions 11

2.1 Parallel $\mathrm{I} / \mathrm{O}$ model . . . . . . . . . . . . . . . . . . . . . . 12

$2.2 \mathrm{I} / \mathrm{O}$ models $\ldots \ldots \ldots \ldots \ldots$

2.3 Terminology . . . . . . . . . . . . . . . . . . . . . 19

2.3.1 What is fair in parallel I/O? . . . . . . . . . . . . 19

2.3.2 Some Useful Terms . . . . . . . . . . . . . . . . . . 21

\section{Optimal Scheduling and NP completeness 23}

3.1 Scheduling Multiple Reference Strings . . . . . . . . . . . . . . . . 24

3.2 NP-Completeness . . . . . . . . . . . . . . . . 26

3.3 Optimal Algorithm for restricted case . . . . . . . . . . . . . . . 34

3.4 Optimal Static Memory Allocation for the PDSB Model . . . . . . . . . . 37

3.5 Approximate Algorithms . . . . . . . . . . . . . . . . . 41 
3.5.1 Static Priority Assignment . . . . . . . . . . . . . . 41

3.5.2 Merge and Schedule . . . . . . . . . . . . . . 42

3.6 Related work $\ldots \ldots \ldots \ldots \ldots \ldots$

4 Scheduling with Fairness/Weighted-QoS 45

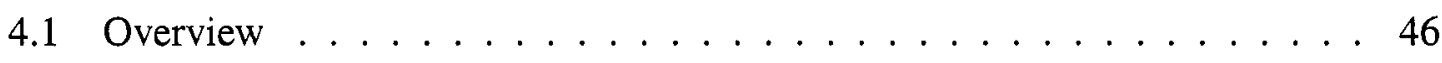

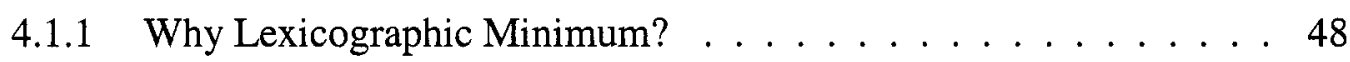

4.1.2 Mapping Disk Scheduling to a Flow problem . . . . . . . . . 48

4.1.3 Why maxflow, bipartite matching algorithms don't work? . . . . 51

4.2 Local fairness . . . . . . . . . . . . . . . . . . 54

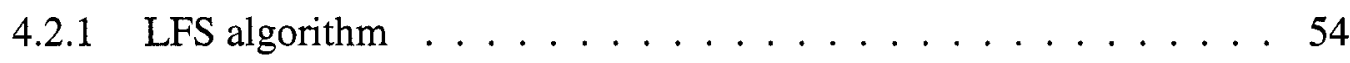

4.3 Global Fairness and QoS Scheduling . . . . . . . . . . . . . . 57

4.3.1 Weighted allocation $\ldots \ldots \ldots \ldots$

4.4 Simulation Results . . . . . . . . . . . . . . . . . . . 61

4.4.1 Random model . . . . . . . . . . . . . . . . . . . . 63

4.4 .2 Skewed Input model $\ldots \ldots \ldots 5$

4.4 .3 Work conservation . . . . . . . . . . . . 68

4.5 Related work . . . . . . . . . . . . . . . . . 68

5 Conclusions and Future Work $\quad 72$

5.1 Future Work . . . . . . . . . . . . . . . . . 72

$\begin{array}{ll}\text { Bibliography } & 74\end{array}$ 


\section{Illustrations}

2.1 Parallel I/O model . . . . . . . . . . . . . . . . . . 16

2.2 PDPB - partitioned disks and memory buffer . . . . . . . . . . 17

2.3 SDPB - shared disks and partitioned memory buffer . . . . . . . . . . 18

2.4 SDSB: shared disk and shared memory buffer . . . . . . . . . . . . . . . . 19

2.5 IO level fairness . . . . . . . . . . . . . . . . . 20

2.6 Block level fairness . . . . . . . . . . . . . . . . . . . . 21

2.7 Global lookahead window . . . . . . . . . . . . . . . 22

3.1 Individual schedules for reference strings . . . . . . . . . . . 29

3.2 Possible schedule with length $2 w B \ldots \ldots . \ldots \ldots$

3.3 Individual schedules for shared memory case . . . . . . . . . . . 33

3.4 Possible schedule with length $2 \mathrm{w} \alpha-(\mathrm{M}-1), \alpha=\mathrm{B}+\mathrm{M}-1 \ldots \ldots$

3.5 Possible paths with two strings . . . . . . . . . . . . . . 36

3.6 Possible paths and space requirement with three strings . . . . . . . . . 38

3.7 Simple static allocation algorithm for two strings . . . . . . . . . . . . 39

$3.8 O(L \log M)$ algorithm for two strings $\ldots \ldots \ldots \ldots$

3.9 Convergence in $\log (\mathrm{M})$ steps $\ldots \ldots \ldots$. . . . . . . . . . . 40

$3.10 \mathrm{Bad}$ case for Static priority Assignment Heuristic . . . . . . . . . . 42

3.11 Schedules created by Static Priority Assignment Heuristic . . . . . . . . 43

4.1 Mapping from disk scheduling to flow . . . . . . . . . . . . 50

4.2 A pathological case for Iterative bipartite matching $\ldots \ldots \ldots$ 
4.3 A pathological case for maxflow algorithm . . . . . . . . . . . 53

$4.4 \mathrm{O}((n+D) n D)$ algorithm for local fair scheduling $\ldots \ldots \ldots$

$4.5 \mathrm{O}((n+D) n D)$ algorithm for global fair scheduling . . . . . . . . . . . 60

$4.6 \mathrm{O}((\mathrm{n}+\mathrm{D}) \mathrm{nD})$ algorithm for weighted allocation $\ldots \ldots \ldots . \ldots 62$

4.7 Output of LFS for 5 strings . . . . . . . . . . . . . . . . 64

4.8 Output of GFS for 5 strings . . . . . . . . . . . . . . . . . . 64

4.9 WeAB with weights $0.4,0.3,0.2,0.1 \ldots \ldots \ldots 5$

4.10 LFS algorithm for skewed inputs . . . . . . . . . . . . . . 66 66

4.11 Fair allocation by GFS (skewed) . . . . . . . . . . . . . 67

4.12 Weighted allocation by $\mathrm{WeAB}($ skewed $) \ldots \ldots \ldots$. . . . . . . 68

4.13 GFS:blocks scheduled per IO vs D . . . . . . . . . . . . . . 69

4.14 GFS:total blocks scheduled in $n$ IOs vs $D^{*} \mathrm{~N} \ldots \ldots$. . . . . . . . . . . 69 


\section{Chapter 1}

\section{Introduction}

Storage requirements of organizations are increasing at a pace faster than ever before. The growing volume of data has placed a huge burden on organizations in terms of reliable, secure, and efficient maintenance of data. The need for data sharing and the economies of scale provide an incentive for companies to seek centralized storage solutions. While large organizations may be able to handle the costs associated with maintaining large and complex in-house storage centers, smaller companies may not be able to afford to maintain and administer such large storage systems.

One trend to reduce the burden of storage management and facilitate sharing is the notion of a Storage Service Provider (SSP). The SSP may operate at the level of a single organization handling storage requirements of different departments, or may be a larger entity providing services to different organizations. As suggested in [Wil01], data centers with a capacity of the order of hundreds of terabytes and transfer rates of tens of gigabytes per second might be common in the near future. Owing to the complexity of such systems, it is crucial to have automated mechanisms for resource allocation in order to be able to provide the desired levels of service to different application workloads. These workloads might have different $\mathrm{I} / \mathrm{O}$ characteristics in terms of their $\mathrm{I} / \mathrm{O}$ rates (IOs per second), and their required response times. To provide even rough guarantees on the performance of an application, it is necessary to provide mechanisms to isolate different workloads from each other, so that performance of one does not degrade the performance of the others. This is not an easy task, due to the complexity of the system and the unpredictable and bursty nature of many I/O workloads. The problem of I/O scheduling to provide a degree of faimess or Quality of Service (QoS) is an important and challenging problem. 
The increasing gap between processing speed and disk I/O latency have made Amdahl's law even more apparent in today's systems. Although the size of main memory is increasing, the amount of data processed by many applications such as scientific computations, seismic data analysis, image visualization and streaming video is too large to be kept entirely in main memory. Such applications depend heavily on I/O system throughput and quality of service to run efficiently. Despite tremendous advancements in disk technology that have resulted in unprecedented density, cost, and speed improvements, the access latency of a disk results in I/O performance that is well short of that required by today's data-centric applications. Even the latest disks have an average seek time close to $5 \mathrm{~ms}$ and average rotational delay of 2 to $3 \mathrm{~ms}$. Also the effective bandwidth served is usually much less than their maximum capacity, which is about 35 to $40 \mathrm{MB} / \mathrm{sec}$. As a consequence, parallel I/O systems consisting of multiple disks connected with high-bandwidth interconnect are the norm in large data centers and supercomputing installations. Traditional bus-based architectures are being supplanted by loosely-connected networked storage using dedicated Storage Area Networks (SANs) with gigabit transfer rates.

In addition to faster hardware and improved I/O organization, software-driven scheduling is used to improve the performance of a storage system. Low-level schedulers with detailed knowledge of disk geometry arbitrate the sequence of disk-arm movements to minimize the latency of a sequence of requests. At the system level, asynchronous $1 / O$ schemes are often employed to reduce the time that an application has to wait for an $I / O$ to complete. In asynchronous $\mathrm{I} / \mathrm{O}$ schemes, the application initiates a request for a disk block before it is actually required, and continues execution while the $\mathrm{I} / \mathrm{O}$ is in progress. If appropriately timed, the block would have been fetched and available in a memory buffer by the time the application actually needs the block. Asynchronous I/O provides a limited form of prefetching, and improves performance by overlapping CPU and I/O operations. However the benefit diminishes as the gap between $\mathrm{L} / \mathrm{O}$ and CPU speed increases.

A more scalable way to mask the $\mathrm{I} / \mathrm{O}$ latency is to use prefetching to exploit the high bandwidth of multiple disk systems. By fetching data from multiple disks in parallel and 
buffering them in main memory the high $\mathrm{I} / \mathrm{O}$ bandwidth can be translated to low access latency. Clearly the gains possible depends on the distribution of data across the disks, and amount of memory buffer available for prefetching.

In a multiple-disk parallel I/O system, data is distributed among the disks to enable simultaneous parallel access. Individual applications can potentially speed up their I/O by fetching data in parallel from multiple disks and buffering the data in memory until they are required. The benefits of prefetching depend on several factors such as the temporal distribution of disk accesses, the amount of buffer memory available to smooth out the uneven distributions of requests, and the amount of lookahead available in order to perform non-speculative prefetching. If the accesses are spread uniformly across the disks, then a small amount of buffer memory is sufficient to obtain high disk utilization; however if the requests are skewed so that at any time only a small subset of the disks are requested, then a large amount of buffer will be needed to compensate for the load imbalance. The second factor, lookahead, refers to the amount of knowledge about future accesses that are available at any time. The amount of lookahead also imposes limits on the prefetching depth. When the load is balanced across the disks, just a small amount of lookahead is sufficient to achieve good disk parallelism, while skewed accesses require large amounts of lookahead that extend across the current hot spot activity. A substantial body of work exists on the problem of prefetching from multiple disks, dealing both with issues of access prediction and scheduling at the system level $\left[\mathrm{PGG}^{+} 95, \mathrm{KJ} 99, \mathrm{WPA}{ }^{+} 03\right]$, as well as scheduling algorithms and their analysis [PSV94, CFKL95, KV99a, AGL00, BKVV00, KK00, KV01, HSV01, KV02, KV, RVV04]. Many of the issues related to scheduling of a single workload are fairly well understood at this time, although some open problems remain. A more detailed discussion is presented in Section 1.3.

When several concurrent applications are simultaneously sharing the $1 / O$ system, the scheduling of the I/Os becomes more complicated. Two issues of concern in this situation are maximizing the utilization of the disk resources by multiplexing the bandwidth among the concurrent tasks, and avoiding starvation of individual tasks. Even when con- 
sidered separately the problems are challenging. For instance, in contrast to the case of a single request stream for which optimal scheduling algorithms have been found [KV99a, $\mathrm{KV01,HSV01,KV02,KV],} \mathrm{the} \mathrm{problem} \mathrm{of} \mathrm{maximizing} \mathrm{the} \mathrm{disk} \mathrm{bandwidth} \mathrm{utilization} \mathrm{while}$ scheduling a set of $\mathrm{n}, n>1$, request streams is shown to be NP-complete in chapter 3 . Besides being computationally intractable and requiring a priori knowledge of the entire set of request streams, such a minimal-length schedule may still be unattractive from the viewpoint of fairness in the allocation of the disk bandwidth to individual workloads.

\subsection{Overview of the Thesis}

In the remainder of this chapter we summarize the major contributions of the thesis, and discuss extant related work. Chapter 2 introduces the model of parallel I/O considered in this thesis. Several different I/O organizations are defined and scheduling problems arising in those contexts are identified. The definitions of various terms and the issues in $\mathrm{I} / \mathrm{O}$ scheduling are discussed in greater detail.

Chapter 3 formally defines the problem of scheduling multiple request sequences and shows it to be NP-complete. In Section 3.3, we consider special cases of the scheduling problem. We show that dynamic programming techniques can be used to construct a minimum-length schedule under the restricted condition of a memory buffer of one block per request sequence. Section 3.4 presents an algorithm for statically allocating the memory buffer to various request sequences in order to minimize the maximum I/O schedule length among the different request sequences. We also consider some simple heuristics for efficient scheduling in section 3.5. Although their worst-case performance is no better than that obtained by naive scheduling strategies, their practical performance may be better. We identify worst-case instances when these heuristics fail to be efficient.

In Chapter 4 we switch our attention to providing fair or differentiated service to different request sequences or flows. Following an overview of the issues, Section 4.1 presents a model for fair block allocation problem and defines faimess metrics. We differentiate between local fairness that attempts to provide fair allocation on an instantaneous basis, and 
global fairness that attempts to provide fair allocation averaged over a long time window. To model differentiated service we use the notion of weighted allocation, to provide bandwidth proportional to the weight of a request sequence. We map the bandwidth allocation problem to that of finding the lexicographically minimum allocation vector. A resource allocation graph tracks the resource constraints among requests and disks. Fair allocation is achieved by transforming the problem to traversing this bipartite graph. In Section 4.1.3 we show that despite similarity with other problems on bipartite graphs, the known algorithms of iterated maximum bipartite matching, and Ford Fulkerson's max-flow algorithm cannot be directly applied to the problem at hand. We present our algorithm, LFS, for achieving local fairness and its correctness in Section 4.2. Two other algorithms, GFS and WeAB, are presented in Section 4.3. These algorithms are used to provide global fairness and differentiated services respectively. Simulation results validate the utility and efficiency of our algorithms in achieving fairness and differentiated service; these are presented in sec-

tion 4.4. Chapter 4 concludes with an overview of related work dealing with faimess and QoS in disk scheduling, network switches, and wireless transmission.

In Chapter 5, we conclude with some ideas for future research.

\subsection{Major Contributions}

The major contributions of this thesis include the following:

- We introduce four I/O organizations based on the partitioning of disks and memory buffer among different request sequences.

- For shared disk I/O organizations, we investigate the offline problem of statically maximizing throughput or obtaining the minimum-length schedule for multiple request sequences, and show that it is NP-complete.

- We present an optimal static algorithm for obtaining the minimum-length schedule under a restricted case, when each sequence has a memory buffer of size 1 
block. The algorithm runs in $\mathrm{O}\left(L^{n}\right)$ time and $\mathrm{O}\left(L^{n-1}\right)$ space where $\mathrm{L}$ is the maximum length of a string and $n$ is the number of sequences.

- We present some scheduling heuristics. While these have a worst case bound no better than that obtainable by trivially scheduling reference strings sequentially one after the other, they may be useful in practice. We identify bad cases for heuristics where they reach their worst case bounds.

- For partitioned disk I/O organizations, we investigate the problem of statically allocating the buffer memory of size $M$ blocks among $n$ request sequences so as to minimize the maximum number of $\mathrm{V} / \mathrm{O}$ 's done for any sequence. Our algorithm runs in $\mathrm{O}\left(L n(\log M)^{\log n}\right)$ time, and obtains the optimal memory allocation. Here $L$ is the maximum length of a request sequence.

- We define the fairness metrics for local and global fairness, and differentiated service in parallel I/O systems.

- We present novel algorithms to guarantee local and global fairness in the presence of resource contention, where individual requests are directed to specific disks. The algorithms run in $O((n+d) E)$ time and are work conserving, where $n$ is the number of request sequences, $d$ is the number of disks and $E$ is the number of simultaneous requests being arbitrated. Since only one request from any request sequence for a particular disk need be considered $E$ is upper-bounded by $n d$.

- We present a generalized, novel algorithm for QoS scheduling to provide differentiated service to different request sequences. The amount of disk bandwidth allocated to each sequence is proportional to the weight assigned to it. It runs in $\mathrm{O}((n+d) E)$ time and is work conserving. 


\subsection{Previous Work}

The work presented in this thesis draws its inspiration from a large amount of previous work in the areas of parallel I/O systems and QoS techniques in networks.

\subsubsection{Parallel Disk Scheduling}

Recent formal work on prefetching in single-disk systems has focused on overlapping I/O with computation. A stall model of sequential I/O incorporating computation time was introduced by Cao et. al. in [CFKL95]. They considered the combined problem of prefetching and caching for a single disk, and showed bounds on the usefulness of aggressive prefetching in reducing the total elapsed time. In the same model, a linear-programming based polynomial time algorithm for minimizing the elapsed time was presented by $\mathrm{Al}$ bers, Leonardi, and Garg in [AGL00]. For the particular objective function they proved that the optimal solution of the linear program is integral, thereby settling the question of polynomial-time optimality raised in [CFKL95].

Early formal work in parallel I/O focused mainly on designing algorithms and datastructures for particular applications; for example external merging and sorting [PSV94, VS94, ZL96, BGV96, KLLV02]. The more general problem of prefetching for read-once reference strings in the parallel disk model was studied by [Kal97, BKVV00]. The special case when the lookahead matches the next buffer-load, that is $L=m$, was considered; it was shown that the competitive ratio of any algorithm with $m$-block lookahead is $\Omega(\sqrt{D})$, and a simple algorithm matching the bound was presented. However having lookahead equal to the size of the buffer is intrinsic to the algorithm and there is no simple generalization which uses extended lookahead. Prefetching schemes based on randomized allocation of data to disks were analyzed in [PSV94,KV99b]. Prefetching algorithms that exploit randomization and replication of data were presented and analyzed in [SEK00].

In [KV99a], Kallahalla and Varman presented the first polynomial time algorithm for optimal offline scheduling of read-once reference strings. Since then the results have been expanded in two directions. In subsequent papers by these authors [KV01, KV02], and in 
the Ph.D. dissertation of Kallahalla [Kal00], they considered the more generalized problem of simultaneous prefetching and caching in the parallel disk model, by considering reference strings with arbitrary repetitions of blocks. An algorithm to construct the optimal offline schedule was presented: this algorithm requires $O(n \log n)$ time [Kal00, KV01, KV02]. When applied to the special case of a read-once reference string (no repetitions) the complexity of the algorithms is linear in the size of the reference string. Analysis of on-line read once scheduling is presented in [KV].

Independently, Hutchinson, Sanders and Vitter [HSV01] explored an elegant and interesting duality between prefetching (reading) and writing in the parallel disk model, and showed how schedules for prefetching in the read-once parallel I/O model can be obtained by first obtaining a schedule for writing all the blocks in the reversed reference string. This approach also leads to a linear time optimal offline scheduling algorithm for read-once reference strings.

Very recently, Shah, Varman and Vitter [RVV04] have examined on-line algorithms for the combined prefetching and caching problem, and explored the use of resource augmentation to study the trade-offs implied by partitioning memory between prefetching and caching.

Other formal studies have looked at the joint problem of prefetching and caching general reference strings (with repetitions) in other parallel I/O models. A parallel disk adaptation of the stall model of [CFKL95] was introduced by Kimbrel and Karlin in [KK00]. A sophisticated analysis of an offline approximation algorithm was presented, and the algorithm was shown to be efficient with respect to elapsed time, for typical system parameters (memory size, number of disks, and ratio of I/O to computation time). A distributed-buffer system, a variant of the parallel disk model where each disk has its private buffer, was studied in [VV99] and an optimal offline prefetching and caching algorithm was presented. 


\subsubsection{QoS Scheduling}

For fair scheduling, FCFS was the first algorithm that was explored to provide best effort service to all the requests without favoring or starving a stream. However, it was noted that serving requests in order of their arrival resulted in unacceptable disk utilization due to the large seek and rotational delays. Many approaches such as Shortest Seek Time First (SSTF), Shortest Seek Time First (STTF) have been proposed to minimize seek and rotational delays. Slight variants such as CSCAN and LOOK have been proposed to avoid starvation caused by the greedy approaches that minimize seek and rotational delays. Haritsa and Pradhan [HP95] showed that the traditional algorithms used for single disk scheduling can be unfair, and they proposed techniques to improve fairness by positioning the disk head in a certain way whenever the disk is idle. There is always a tussle between improving the efficiency and fairness of scheduling techniques, as these two goals are often contradictory to each other.

Many algorithms like Cello [SV98], YFQ [BBG $\left.{ }^{+} 99\right]$ have been proposed for QoSdriven single disk scheduling. These approaches address the situation when multiple applications with different performance requirements concurrently access the disk. For example, file transfer applications such as ftp, interactive best- effort applications such as word processors, and real-time applications such as streaming audio and video, that have different access characteristics and performance requirements, may need to be simultaneously serviced by a disk. Cello [SV98] suggests a two level scheduling framework where a scheduler exists for each type of application at the first level, and all these first level schedulers put requests into a common queue, depending on the application's performance requirement. This common queue is then scheduled for efficiency by the second level scheduler. On the other hand, YFQ [BBG $\left.{ }^{+} 99\right]$ assigns a virtual start and finish times to each of the requests based on their requirements, and tries to approximate the well-studied generalized processor sharing algorithm (GPS) proposed for fair scheduling.

There is a considerable amount of literature on resource scheduling in datagram networks [Zha90, Go194, GVC96, BZ96, BZ97]. Most of these approaches are directed to- 
wards approximating the ideal GPS algorithm to make it implementable, and differ based on their achievable fairness guarantees, delay bounds and cumulative service provided to the flows. Recently some approaches such as Façade [CLA03], Interposed Proportional sharing [JCK04], and Stonehenge [HPC04], have been suggested for multiple resource scheduling in storage systems. Façade [CLA03] provides a virtualization approach to fulfill the Service Level Objective (SLO) of independent workloads accessing a storage system by doing EDF (earliest deadline first) scheduling. Façade provides a dynamic trade-off between the IO rate and average latency, by real-time scheduling and feedback-based control of disk queue lengths. Stonehenge [HPC04] proposes a virtual clock- based two-level disk scheduling architecture. At the first level every request goes to a central controller that determines a deadline for the request, and at the second level a CVC disk scheduler is used at each disk. CVC maintains dual queues, one for handling QoS deadlines and another to maximize disk utilization based on the slack available in meeting deadlines. Interposed proportional sharing algorithms are suggested in [JCK04]; these assign virtual start and finish times to requests based on their cost, weight of the client queue and arrival time. They show their algorithm to be fair as well as work conserving. 


\section{Chapter 2}

\section{Models and Definitions}

In this chapter we introduce the model of parallel $1 / O$ that we are going to use throughout the thesis. Although the storage systems today are very complex and involve many sources of contention, we construct an abstract model that hides many physical details, and considers the issues of parallel $\mathrm{I} / \mathrm{O}$ at a higher level. In our idealized abstraction issues such as variability in disk delays and interconnect scheduling are not explicitly considered. However, lower-level optimizations can be made to the schedules to further improve the performance.

The workload presented by a single application in an $\mathrm{I} / \mathrm{O}$ system will be abstracted as a reference string which is denoted by $R$. A reference string is an ordered sequence of requests made to the $\mathrm{I} / \mathrm{O}$ system. For example, $R=A_{1} B_{1} A_{2} A_{3} B_{2} B_{3} C_{1} C_{2} B_{4}$, is a possible reference string in a three-disk system, where the alphabets $\mathrm{A}, \mathrm{B}$, and $\mathrm{C}$ denote different disks, and subscripts denotes different block numbers. If all the blocks in a reference string are unique, then it is referred to as a read-once reference string. On the other hand, if there are several references to the same block in the reference string, then it is referred to as a general or read-often reference string. The key characteristic of a read-once reference string is that there is no value in caching a block once it has been referenced by the application. In contrast, in a read-often reference string a block should not be necessarily be discarded after being referenced since it may be requested again in the near future. In this case, caching the block between references could save an additional I/O. In this work, we will consider both types of reference strings.

At any time, multiple applications, each with their own reference string, might be accessing the I/O system. We use $n$ to denote the number of such concurrent independent 
reference strings or applications. We show that the problem of obtaining a minimum length schedule for $n$ consecutive applications is NP-complete for read-once reference strings. Later on we provide algorithms for weighted-fairness considering reference strings with no restriction on the types of requests.

In the rest of the chapter we will present our model for a parallel $\mathrm{I} / \mathrm{O}$ system. We will also describe the information required by scheduling algorithm for efficient prefetching and buffering of disk blocks.

\subsection{Parallel I/O model}

A parallel I/O system consists of $d$ independent disks $\mathcal{D}=\left\{D_{1}, D_{2}, \cdots, D_{d}\right\}$. Data is stored on the disks in units of blocks; a block is the unit of access from a disk. In each $\mathrm{I} / \mathrm{O}$ one block can be fetched from each disk. Hence a model with $d$ disks can provide a parallelism of at most $d$. However a speedup of $d$ is not achieved in practice due to skewed access patterns that direct requests to only a subset of the disks at any time, or because memory buffer constraints prevent fetching from some disks. At each $\mathrm{I} / \mathrm{O}$ step one or more blocks requested by the concurrent workloads are fetched into the buffer. An application consumes blocks in the order specified by its reference string, freeing up buffer space following the reference to a block. A disk remains idle if no block is fetched from it in the current $\mathrm{I} / \mathrm{O}$.

The blocks fetched from the disks may be buffered in internal memory buffer until they are required. The presence of the buffer allows applications to prefetch blocks and reduce the I/O latency. Prefetching is defined as the accessing of a block and storing it in memory buffer before the block is requested by the application; later the block can be serviced directly from the memory buffer when requested. Thus prefetching helps in reducing the total number of I/O's by utilizing the idle disk bandwidth to overlap I/O requests. However prefetching in a multiple-disk system needs to be done carefully, as simple greedy prefetching can result in poor performance [BKVV00, KV99a].

An I/O schedule consists of a sequence of parallel I/O steps: in each step at most one block from each of the $d$ disks is accessed. Table 2.1 shows an example of a greedy 


\begin{tabular}{|c|c|c|c|c|c|c|c|c|c|}
\hline IOs $\rightarrow$ & 1 & 2 & 3 & 4 & 5 & 6 & 7 & 8 & 9 \\
\hline Disk A & $A_{1}$ & $A_{2}$ & $A_{3}$ & $A_{4}$ & $A_{5}$ & $A_{6}$ & $A_{7}$ & - & - \\
\hline Disk B & $B_{1}$ & $B_{2}$ & $B_{3}$ & - & - & $B_{4}$ & $B_{5}$ & $B_{6}$ & $B_{7}$ \\
\hline Disk C & $C_{1}$ & $C_{2}$ & - & - & - & - & - & - & - \\
\hline
\end{tabular}

Table 2.1 : Greedy IO schedule

schedule for the reference string $R$ shown below. The example assumes there are three disks $A, B$, and $C$, and that the size of the buffer $M$ is 6 blocks. The notation $A_{i}\left(B_{i}, C_{i}\right)$ means a request for block $i$ from disk A (respectively $\mathrm{B}, \mathrm{C}$ ).

$$
\mathcal{R}=A_{1} A_{2} A_{3} A_{4} A_{5} B_{1} B_{2} A_{6} B_{3} B_{4} B_{5} A_{7} C_{1} C_{2} B_{6} B_{7}
$$

In step 1 of Table 2.1, blocks $A_{1}, B_{1}$ and $C_{1}$ are fetched from the three disks respectively. $A_{1}$ is consumed, and a request for the next block $A_{2}$ is made. Since there are four free blocks in the buffer at this time, the system will prefetch the next block from disks $B$ and $C$ along with $A_{2}$ in the next $\mathrm{I} / \mathrm{O}$ step. On making the reference to $A_{3}$, the buffer holds 4 blocks; hence it cannot prefetch blocks from both $B$ and $C$, but must choose to prefetch from one or the other of the disks. A greedy in-order prefetching scheduling algorithm will fetch $B_{3}$ in preference to $C_{3}$ since it occurs earlier in the reference string. Continuing in this manner, we obtain the schedule of length 9 to service the entire reference string. In contrast, Table 2.2 shows the optimal-length schedule for this reference string consisting of 7 parallel I/O steps.

The efficiency of a schedule is measured by the $1 / O$ schedule length. The throughput is measured as the ratio of the total number of blocks fetched to the number of parallel $\mathrm{I} / \mathrm{O}$ steps needed. Minimizing the number of I/O's for a set of reference strings is equivalent to maximizing the throughput.

In [BKVV00] it was shown that the length of the schedule created by greedy in-order prefetching is bounded by $\Theta(\sqrt{d})$ times the length of an optimal schedule, and that the 


\begin{tabular}{|c|c|c|c|c|c|c|c|}
\hline IOs $\rightarrow$ & 1 & 2 & 3 & 4 & 5 & 6 & 7 \\
\hline Disk A & $A_{1}$ & $A_{2}$ & $A_{3}$ & $A_{4}$ & $A_{5}$ & $A_{6}$ & $A_{7}$ \\
\hline Disk B & $B_{1}$ & $B_{2}$ & $B_{3}$ & $B_{4}$ & $B_{5}$ & $B_{6}$ & $B_{7}$ \\
\hline Disk C & - & - & - & - & - & $C_{1}$ & $C_{2}$ \\
\hline
\end{tabular}

Table 2.2 : Optimal IO schedule

bound is tight. However, if the lookahead available is limited, less than or equal to $M$, then this is the optimal scheduling strategy. The problem of obtaining the optimal offline schedule and online scheduling for larger lookaheads were considered in [KV99a, KV] respectively.

In our study, we assume that multiple independent reference strings are accessing the storage system simultaneously. The problem of getting an efficient schedule becomes harder due to the contention among strings for a disk. The single reference string situation involved selecting which disk would be provided with a free buffer; in the multiple reference string situation an additional decision of which reference string should get the buffer needs to be made as well. To understand the complexity in scheduling multiple reference strings, consider a simple scenario with 2 reference strings $R_{1}$ and $R_{2}$, accessing 2 disks concurrently and a buffer of size 3 for each string. Each reference string attempts to greedily fetch its blocks in order. At each point, there might be a contention for disks among the reference strings as shown in Table 2.3 , and the arbitration can result in a considerable difference in the length of the overall schedule obtained.

$$
\begin{gathered}
R_{1}=A_{1} A_{2} A_{3} A_{4} A_{5} A_{6} B_{1} B_{2} B_{3} B_{4} B_{5} A_{7} A_{8} A_{9} \\
R_{2}=A_{20} A_{21} A_{22} B_{20} B_{21} B_{22} B_{23} B_{24} B_{25} B_{26}
\end{gathered}
$$

For example consider a schedule where $R_{1}$ is preferred over $R_{2}$, whenever there is a conflict among them for a certain disk. The schedule obtained by using the policy is shown 


\begin{tabular}{|c|c|c|c|c|}
\hline IOs $\rightarrow$ & 1 & 2 & 3 & 4 \\
\hline Disk A & $A_{1} / A_{20} ?$ & $A_{2} / A_{21} ?$ & $A_{3} / A_{22} ?$ & - \\
\hline Disk B & $B_{1} / B_{20}$ & $B_{2} / B_{21} ?$ & - & - \\
\hline
\end{tabular}

Table 2.3 : Contention at each step

\begin{tabular}{|c|c|c|c|c|c|c|c|c|c|c|c|c|c|c|c|c|}
\hline IOs & 1 & 2 & 3 & 4 & 5 & 6 & 7 & 8 & 9 & 10 & 11 & 12 & 13 & 14 & 15 & 16 \\
\hline $\mathrm{A}$ & $A_{1}$ & $A_{2}$ & $A_{3}$ & $A_{4}$ & $A_{5}$ & $A_{6}$ & $A_{7}$ & $A_{8}$ & $A_{20}$ & $A_{9}$ & $A_{21}$ & $A_{22}$ & - & - & - & - \\
\hline $\mathrm{B}$ & $B_{1}$ & $B_{2}$ & $B_{20}$ & $B_{21}$ & - & - & $B_{3}$ & $B_{4}$ & $B_{5}$ & - & - & $B_{22}$ & $B_{23}$ & $B_{24}$ & $B_{25}$ & $B_{26}$ \\
\hline
\end{tabular}

Table 2.4 : IO Schedule when $R_{1}$ is preferred

in Table 2.4. Note that $B_{1}, B_{2}$ are prefetched in memory in first two IOs, but $B_{3}$ cannot be prefetched because no space is available in the memory buffer. A similar reason holds for $B_{22}$, since two buffers are already occupied by $B_{20}$ and $B_{21}$, and the last buffer is needed to fetch $A_{20}$. Hence constraints posed by memory and disks results in a schedule of length 16 in this case. A shorter schedule with 13 IOs is obtained when $R_{2}$ is preferred over $R_{1}$ as shown in Table 2.5. We show this problem of finding the minimum length schedule to be $\mathrm{NP}$-complete later in this thesis.

An example of a parallel I/O system is shown in Figure 2.1. In Figure 2.1, the arrow between the disk array and memory denotes the total throughput from the disk system and the arrows between the memory buffer and individual strings denote the bandwidth

\begin{tabular}{|c|c|c|c|c|c|c|c|c|c|c|c|c|c|}
\hline IOs $\rightarrow$ & 1 & 2 & 3 & 4 & 5 & 6 & 7 & 8 & 9 & 10 & 11 & 12 & 13 \\
\hline Disk A & $A_{20}$ & $A_{21}$ & $A_{22}$ & $A_{1}$ & $A_{2}$ & $A_{3}$ & $A_{4}$ & $A_{5}$ & $A_{6}$ & $A_{7}$ & $A_{8}$ & - & $A_{9}$ \\
\hline Disk B & $B_{20}$ & $B_{21}$ & $B_{1}$ & $B_{2}$ & $B_{22}$ & $B_{23}$ & $B_{24}$ & $B_{25}$ & $B_{26}$ & $B_{3}$ & $B_{4}$ & $B_{5}$ & - \\
\hline
\end{tabular}

Table 2.5 : IO Schedule when $R_{2}$ is preferred 
Disk Array
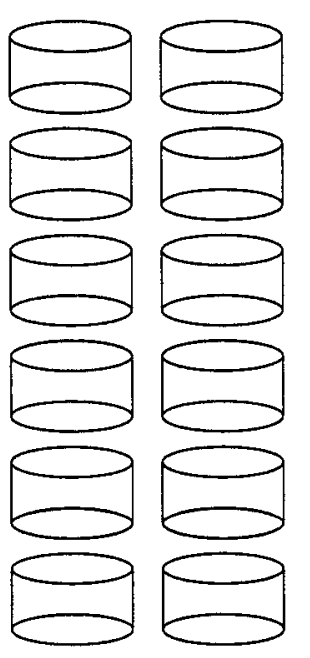
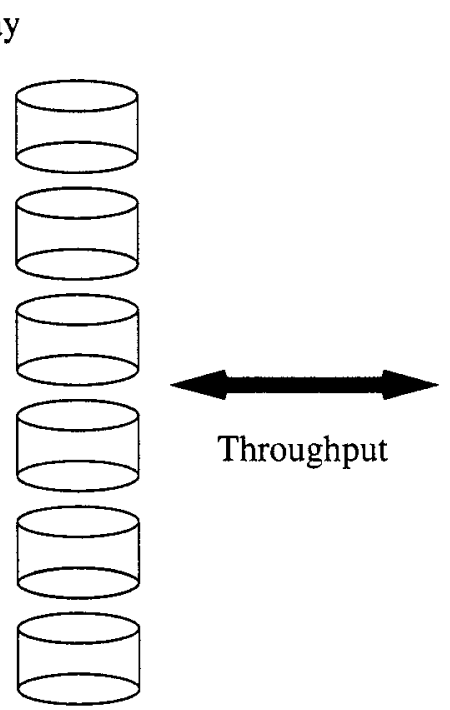

Memory Buffer

Reference Strings

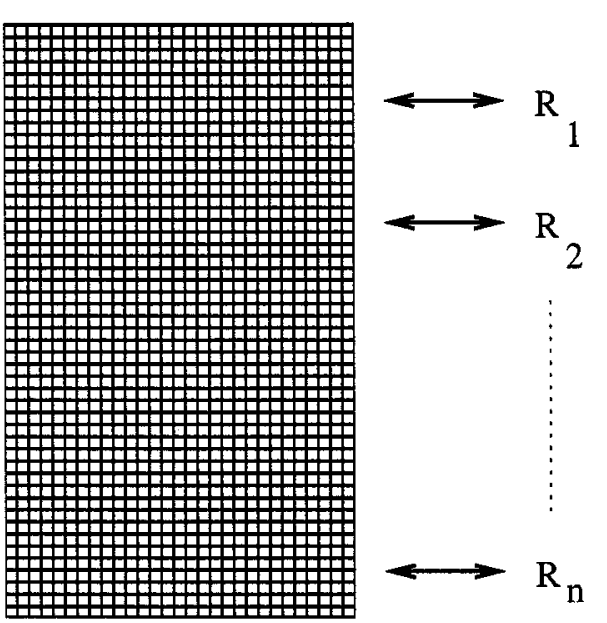

Figure 2.1 : Parallel I/O model

allocated to each string.

\subsection{I/O models}

When multiple resources are shared between various tasks, performance isolation becomes a very challenging problem. Performance isolation is the act of insulating a task's performance from the effect of resource contention by the other tasks. One way to provide performance guarantees is to prevent sharing of resources between tasks. In the context of $\mathrm{I} / \mathrm{O}$ systems, there are several problems with this approach. One is the difficulty in data sharing among different applications. Workloads requiring access to the same data or even to different data stored on the same disks, would either need to run sequentially or would require data sets to be replicated across multiple disk units. Secondly, the approach is insensitive to the variations in buffer requirements of different workloads. As mentioned earlier, well-distributed workloads can work with far less buffer than would be required by a workload with skewed access to the same set of data. Given the difficulty of predicting the storage requirements of workloads, such over-provisioning can lead to solutions that are 


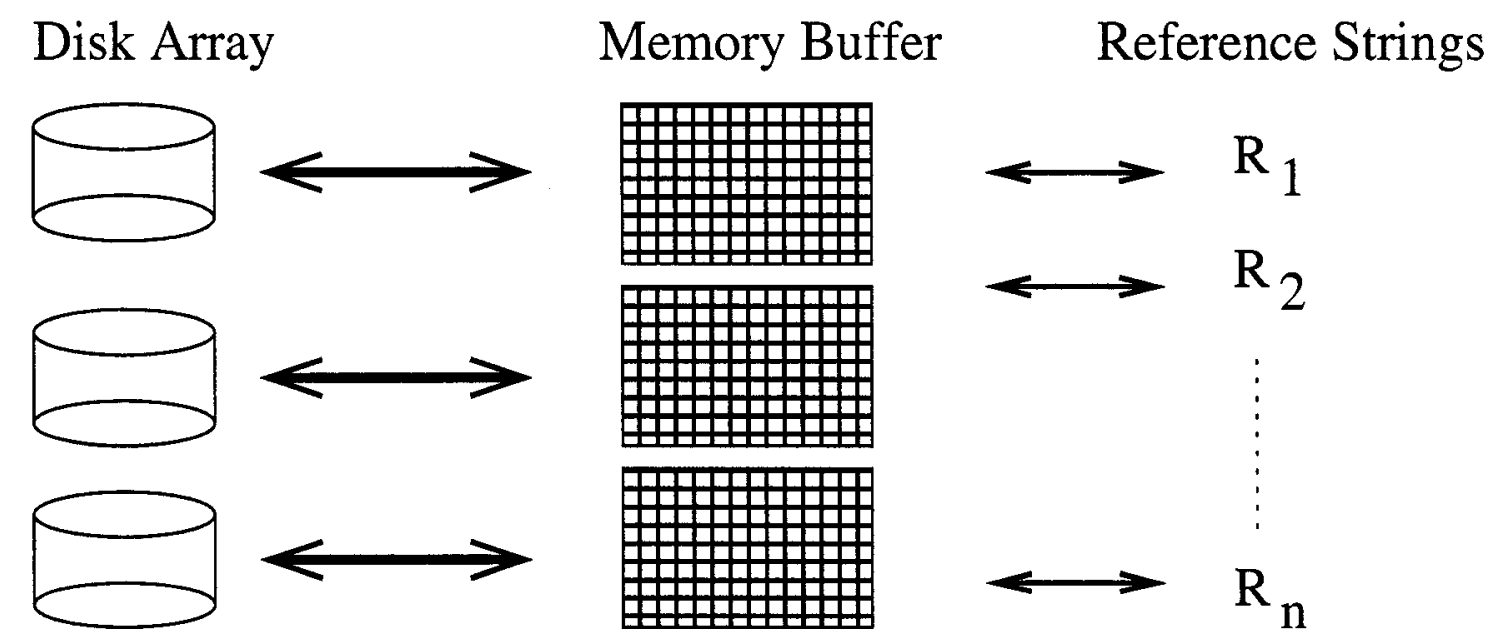

Figure 2.2 : PDPB - partitioned disks and memory buffer

unnecessarily expensive. We consider four classes of systems, that differ in their sharing of buffer memory and disk resources.

The four models that we considered are:

1. PDPB: Partitioned Disk, Partitioned Buffer

2. PDSB: Partitioned Disk, Shared Buffer

3. SDPB: Shared Disk, Partitioned Buffer

4. SDSB: Shared Disk, Shared Buffer

The PDPB model essentially forces workload isolation by spatially partitioning the system into disjoint resource collections. As noted above, there are difficulties in using such an arrangement in a dynamic storage system environment. An example of this configuration is shown in Figure 2.2.

In the PDSB model, the disks are partitioned among workloads so that each reference sting accesses its own set of disks. However, there is a common buffer pool that is shared by all the workloads. Such an organization is most useful in a batched workload environment, where data can be a-priori partitioned into appropriate disjoint data sets. In this situation, 


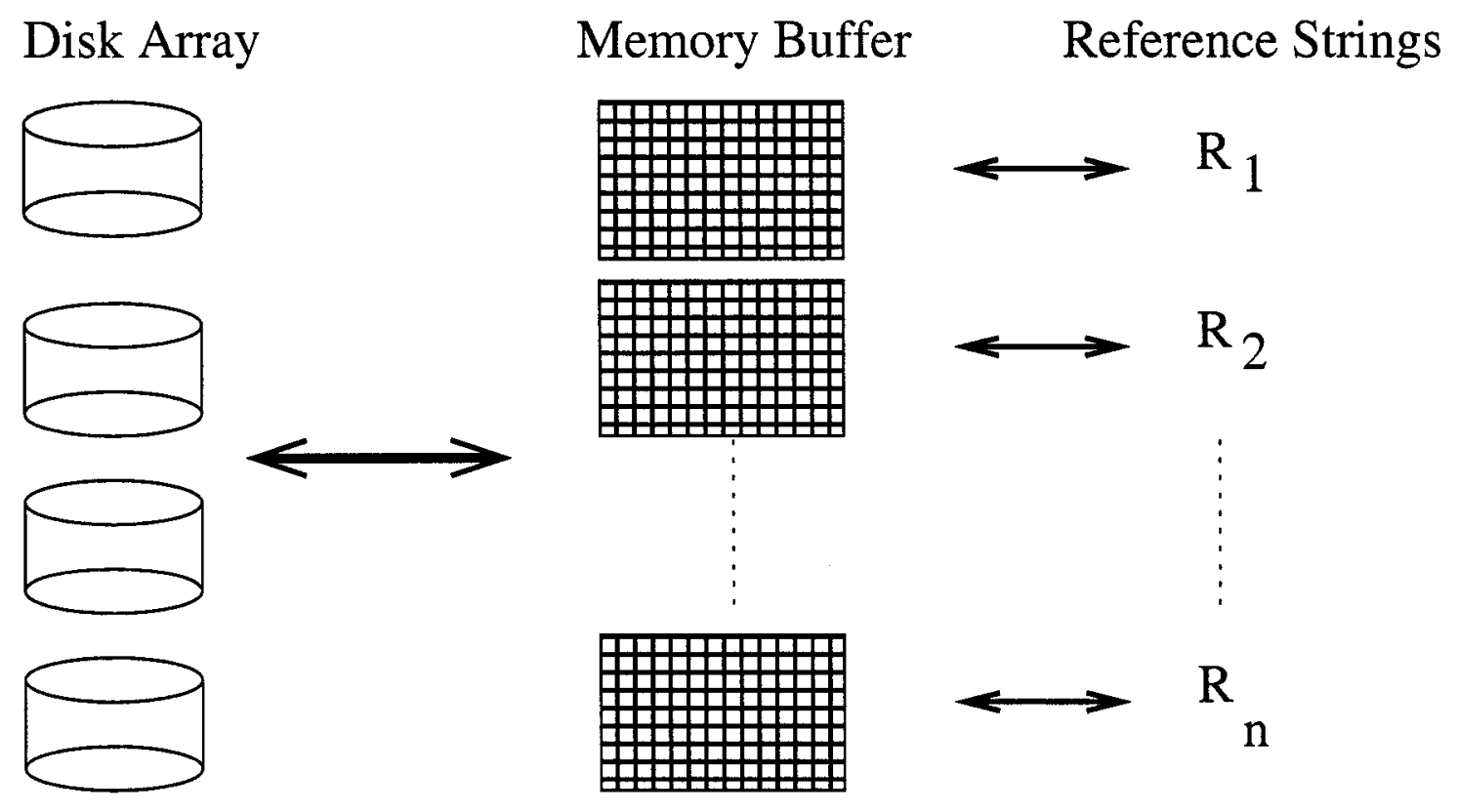

Figure 2.3 : SDPB - shared disks and partitioned memory buffer

we design an algorithm that optimally partitions the memory buffer statically among the workloads so as to minimize the longest schedule.

The SDPB and SDSB models represent the common case where disks are shared among all the workloads. In SDPB, the buffer is partitioned among the workloads. The most common scenario for such an organization is where the buffering is done in individual client nodes and the disks are accessed over a SAN. In the SDSB model, the buffer is logically shared among all workloads. This may be either in the form of a centralized storage buffer, or as has been explored in other works [DAPW94], be made up of distributed shared memory. See Figures 2.3 and Figure 2.4 for these two models. In general, the shared models allow better utilization of resources if scheduled appropriately, but intensifies the problem of providing fairness. 


\section{Disk Array}

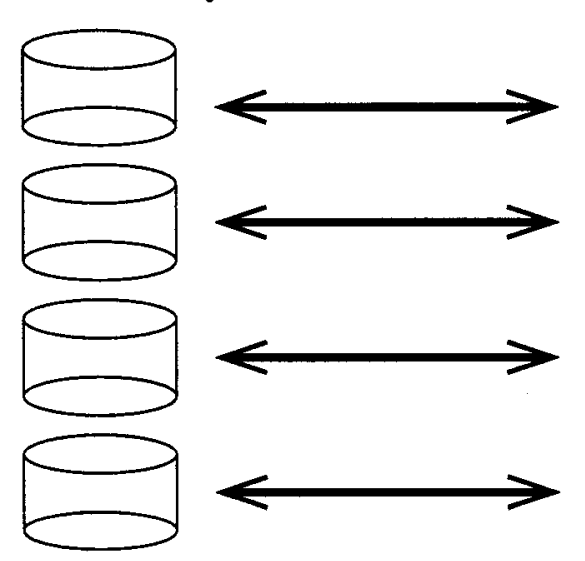

Memory Buffer

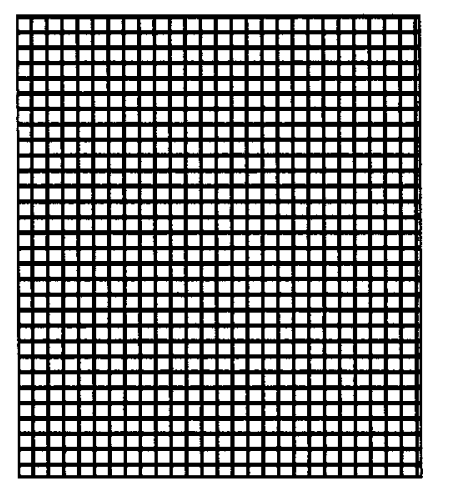

Reference Strings

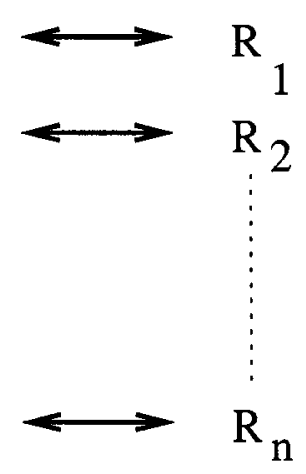

Figure 2.4 : SDSB: shared disk and shared memory buffer

\subsection{Terminology}

We have argued that the problem of faimess is quite significant for a large data center where the amount of shared bandwidth is large, and multiple users with different interests and priorities are paying for the services. Workloads served are often unpredictable and they change with time. Hence a framework is required to support the economic model used by these data centers. Before solving the fairness problem, we need to define the notion of faimess in parallel $\mathrm{I} / \mathrm{O}$ systems.

\subsubsection{What is fair in parallel $\mathrm{I} / \mathrm{O}$ ?}

Fairness in sharing can be defined as the equal distribution of resources among the entities using them. If a single resource is being shared, then it can serve only one of the multiple users at any instant, hence fairness is measured over a certain period of time and there is no concept of mometary fairness. On the other hand, if multiple resources are shared between different users, then the distribution can try to be fair both at each instant as well as over a longer window of time. Parallel I/O falls in the latter category. Let us consider a simple example with three reference strings, three disks (A, B and C) and a memory buffer of size 


\begin{tabular}{|c|c|c|c|c|c|c|c|c|c|}
\hline IOs $\rightarrow>$ & 1 & 2 & 3 & 4 & 5 & 6 & 7 & 8 & 9 \\
\hline Disk A & & & & & & & & & \\
\hline Disk B & $w$ & & & 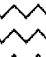 & 1 & & $\approx$ & & . \\
\hline Disk C & & & & $\hat{\gamma}$ & & & & itis & \\
\hline
\end{tabular}

Figure $2.5:$ IO level fairness

$m$ shared by all the strings.

One notion of fairness is to allocate an equal number of IOs to each of the strings, as shown in Figure 2.5. It is very simple to implement but it can lead to poor utilization of resources because a string may not have requests for all the disks in a certain $\mathrm{VO}$, and we chose to idle the disk, stopping others from using that spare bandwidth. This is shown by holes in Figure 2.5 where a request belonging to some other reference string could be scheduled. Another notion of fairness is to allocate equal number of blocks served to each string. In this case, we try to allocate disks evenly among tasks at each $\mathrm{V} O \mathrm{O}$ if possible. Empty slots for which there is no contention can be allocated arbitrarily to increase the system utilization. A sample schedule with block level fairness is shown in Figure 2.6. In this case, we try to allocate disks in an even way in each $\mathrm{I} / \mathrm{O}$. For example we assign one disk to each of the reference strings in IOs 1 and 2. Ocassionaly we might do an unfair local distribution, because of lack of requests from one reference string for some disk. Over a period of time, we attempt to even out these local fluctuations.

Our faimess metric is based on block level fairness and it assumes that each request consumes an equal amount of resource. We only care about requests that are made to the disk system, and every request that is serviced by disk system is counted as an allocation of a unit resource. An average value for request service requirements can be used in cases, where the service requirements are not known a priori (a common scenario in storage systems). We assume that we always have enough memory buffer to store prefetched blocks 


\begin{tabular}{|c|c|c|c|c|c|c|c|c|c|}
\hline $\mathrm{IOs} \rightarrow$ & 1 & 2 & 3 & 4 & 5 & 6 & 7 & 8 & 9 \\
\hline Disk A & & & & & & & & & $m$ \\
\hline Disk B & & & & $\checkmark$ & & 8 & & & 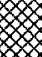 \\
\hline Disk C & & $\infty x$ & 4 & & & & & & 4 \\
\hline
\end{tabular}

Figure 2.6 : Block level fairness

so that none of the disks is left idle if there is a request for that disk in the current lookahead window. An algorithm that fetches from a disk whenever there is some request that can be fetched in any $\mathrm{I} / \mathrm{O}$, is defined as a work conserving algorithm. Although we consider storage systems as a major application, our algorithms can be used in any scenario where multiple resources are shared among users and there are resource constraints posed by different requests.

\subsubsection{Some Useful Terms}

Prefetching is crucial for obtaining good efficiency of any scheduling algorithm using parallel I/O. Some knowledge about future requests can greatly improve the task of buffer management. Even in the case of a single disk, a limited form of prefetching is often done, such as fetching some blocks adjacent to the current request. This works in practice because of the spatial locality in disk requests. This speculative prefetching saves $\mathrm{I} / \mathrm{O}$ time in cases when block sizes are small and the access time is dominated by seek and rotational delays. However, apart from the coalescing of physically close requests prefetching cannot reduce the total number of $\mathrm{I} / \mathrm{O}$ 's needed for a sequence of requests in a single disk case. In contrast, parallel disk system allows simultaneous access to multiple disks overlapping the I/O time for those requests. At each step disks which are not fetching demand blocks, can be utilized to prefetch blocks further down in reference strings. This information about 


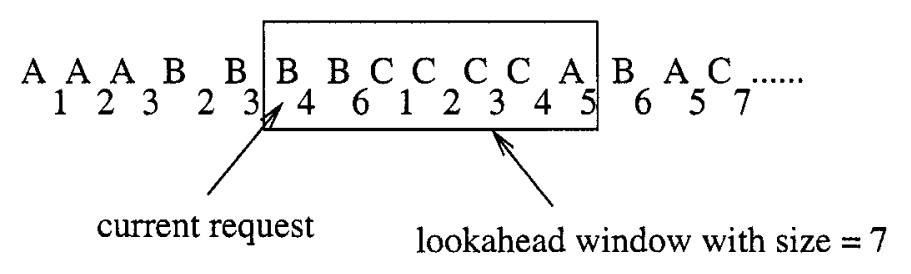

Figure 2.7: Global lookahead window

future accesses is modeled as a lookahead window.

There are two types of lookahead defined in the literature- global and local. In global lookahead we have knowledge of some future blocks from the active reference string. In case of local lookahead, a certain number of future blocks for each disk is known, but the relative order between disks is not known. We will assume global lookahead for each reference string unless otherwise stated. An example of global lookahead with a window of length 7, is shown in Figure 2.7. Alphabets A, B and C denote different disks and subscripts denote block numbers. The block immediately required by the application is known as the demand block and blocks after that form the lookahead window. For example $B_{4}$, that is the demand block in Figure 2.7 and the next 6 blocks form the lookahead window.

We call an algorithm for which the complete reference string is known in advance as an offline algorithm and an algorithm for which only a limited amount of lookahead is available as on-line. Offline cases are more interesting from the theoretical point of view, since they provide a benchmark against which the practical algorithm can be compared.

In chapter 3 of this thesis we try to find offline algorithms to obtain the minimum length schedule when multiple reference strings try to access the disk system. We show that the problem of maximizing throughput is NP-complete in this case. We use the total number of I/O's taken by a schedule to service a set of disk requests as the performance metric. This measure is related to the amount of time spent in servicing all the requests, but ignores the variance in individual operation times due to different seek and rotational latencies, or varying request sizes. 


\section{Chapter 3}

\section{Optimal Scheduling and NP completeness}

In this chapter we consider the problem of scheduling multiple reference strings on the different parallel I/O models discussed earlier. We investigate the construction of I/O schedules that maximize the throughput of the disk system by servicing all the reference strings in the minimum number of parallel $\mathrm{I} / \mathrm{O}$ steps. Such a schedule that requires the minimum number of I/O's to access all the blocks in the reference strings is called an optimal or min-schedule.

Scheduling for a single disk is mainly dominated by issues involved in orchestrating the movement of the disk arm in order to minimize mechanical latencies due to seek and rotational delays. Scheduling strategies attempt to balance the conflicting goals of increased throughput and bounded response times for requests: while throughput can be increased by scheduling physically proximate requests together, this can potentially increase the response time for outlying requests.

In our work, we take a system-level rather than a device perspective of the scheduling problem. The order in which requests are dispatched to the individual disks in a parallel system has a significant effect on the utilization of the system as a whole. Because of skew in the request sequence, the load on different disks varies with time resulting in full utilization of only a fraction of the available disks at any time. As noted in Chapter 2, a high-level schedule attempts to distribute the load as evenly as possible subject to limitations on the buffer size.

We believe that a two-level hierarchical approach to scheduling in parallel I/O systems is a feasible and attractive approach. At the higher-level we construct schedules based on an idealized model of the underlying system, neglecting the disk parameters and lower- 
level optimizations that can be obtained by exploiting the disk geometry. This high-level schedule is used to feed the lower-level schedulers for each disk, which then optimize among the requests in their individual disk queues. For instance, one of the abstractions used in constructing a schedule is the notion of scheduling in rounds: that is in one I/O step, all the requests made to the disks complete before the next round is initiated. A strict implementation of this schedule would suggest that the length of a round is set by the slowest I/O request dispatched in that round. However, the two-level approach does not require this synchronous round-like behavior for the actual scheduling. The round-based schedule provides an ordering of the requests to be dispatched to the disks. This high-level schedule captures long-range optimizations which allow better load balance. The requests dispatched to each disk can be scheduled independently by the disk, without destroying the long-range benefits identified by the high-level schedule. Our scheduling algorithm can be run at a disk array controller to chose the requests to be dispatched to the different disk queues, or as a shin box that sits between all client queues and the storage system.

\subsection{Scheduling Multiple Reference Strings}

We study the problem of constructing a min-schedule for two or more reference strings. Obtaining a min-schedule for a single reference string in the shared-disk model is solvable in linear time for read-once reference strings and with an additional logarithmic factor for read-many reference strings, as shown in [KV01, KV02, KV]. Note that for a single reference string, all four L/O models defined in Chapter 2 are equivalent. In the case of multiple reference strings, two different optimization problems arise in constructing a minschedule for the shared and partitioned disks models respectively. We define these problem formally below.

- Shared Disk Partitioned Buffer Scheduling: The problem is parameterized by several quantities: $n, d, m, L$, and $K$, and will be denoted by $\operatorname{SDPB}(\mathrm{n}, \mathrm{d}, \mathrm{m}, \mathrm{L}, \mathrm{K})$, where: $n$ is the number of read-once reference strings whose data is distributed among a set of $d$ disks $\mathcal{D}=\left\{D_{1}, D_{2}, \ldots, D_{d}\right\}$, a memory buffer of size $M=m \times n$ blocks partitioned 
into individual buffers of size $m$ blocks for each string, $L$ is the maximum length for a reference string, and $K$ is a desired bound on the length of the schedule. The decision problem is to determine if there exists a schedule that requires less than or equal to $K$ V/O steps. The corresponding optimization problem is to actually construct a schedule of minimum length. Note that in a valid schedule, each reference string consumes blocks in the order specified, but there is no restriction on the ordering across strings. Furthermore, each reference string can hold at most $m$ blocks in the buffer at any time, including the current demand block.

- Shared Disk Shared Buffer Scheduling: The problem is parameterized by several quantities: $n, d, M, L$, and $K$, and will be denoted by $\operatorname{SDSB}(\mathrm{n}, \mathrm{d}, \mathrm{M}, \mathrm{L}, \mathrm{K})$, where: $n$ is the number of read-once reference strings whose data is distributed among a set of $d$ disks $\mathcal{D}=\left\{D_{1}, D_{2}, \ldots, D_{d}\right\}$, a memory buffer of size $M$ blocks shared among all reference strings, $L$ is the maximum length for a reference string, and $K$ is a desired bound on the length of the schedule. The decision problem is to determine if there exists a schedule that requires less than or equal to $K \mathrm{I} / \mathrm{O}$ steps. The corresponding optimization problem is to actually construct a schedule of minimum length. Note that in a valid schedule, each reference string consumes blocks in the order specified, but there is no restriction on the ordering across strings. Furthermore, at most $M$ blocks can be present in the buffer at any time, including the current demand block of at least one of the strings.

- Partitioned Disk Shared Buffer Scheduling: The problem is parameterized by several quantities: $n, d, M, L$, and $K$, and will be denoted by PDSB(n,d,M,L, $\mathrm{K}$ ), where: $n$ is the number of read-once reference strings; the data of each string is distributed among a set of disks and the sets of disks used by each string are disjoint. A memory buffer of size $M$ blocks is shared among all reference strings, $L$ is the maximum length for a reference string, and $K$ is a desired bound on the length of the schedule. 
We consider a version of this problem where the buffer is statically partitioned among all the reference strings. Once each string is designated a fixed portion of the buffer it can proceed independently of the rest, since it does not have disk conflicts with the other reference strings. The optimization problem in this case is to find an allocation of buffer to reference strings, so that the maximum-length schedule of the reference strings is minimized.

\subsection{NP-Completeness}

In this section, we present the proofs for NP-completeness of the scheduling problems for the shared disk models. We briefly present an informal description of the complexity class NP and some definitions to help a non-specialist reader in following the proof.

The class NP is a complexity class that consists of the problems that can be verified in polynomial time using a non-deterministic Turing Machine. NP-complete problems are a subset of the hardest problems in NP. The foundation of this class was laid by the famous paper by Stephen Cook [Coo71] in 1971. In that paper Cook proved the NPcompleteness of the satisfiability problem, and introduced the concept of polynomial time reducibility to show the equivalence in difficulty between different problems. In a couple of years, hundreds of problems such as vertex-cover, subset sum, Hamiltonian path and the traveling salesman problem were added to the complexity class NP-complete. It is unfortunate that some of the very common and useful problems belong to this complexity class. If any NP-complete problem can be shown to have a polynomial time solution, then all NP-complete problems will also have one. Despite tremendous efforts by computer scientists over decades this has not been the case, and it is widely believed (though yet unproven) that NP-complete problems do not have a polynomial time solution.

An algorithm is called tractable if it runs in time bounded by a polynomial in terms of the length of the input. In other words, if a problem instance can be encoded in a string of length $n$, then the algorithm should run in time $\mathrm{O}\left(n^{k}\right)$ for some constant $k$.

The encoding a problem is very important for the class NP. In our problem the length 
of input is the sum of lengths of all input reference strings. Furthermore, each reference string will be assumed to be fully enumerated by the sequence of blocks that it accesses. Hence, a reference string with $L$ requests is assumed to require $\Omega(L)$ bits to represent. That is we do not assume any form of compression of the input reference string. For instance, it is conceivable that certain input patterns like requests to sequential block numbers on a disk need not necessarily be enumerated individually but may instead be represented in some compressed form, or some other information-theoretic based compression schemes could be applied to reduce the length of the input. We disallow specially crafted encodings and assume explicit enumeration of the requests. That is each request requires at least a constant number of bits. In practice, each request will require at least $\Omega(\log L)$ bits to encode the disk number and block number within the disk, but this level of representation detail is not needed for our result. This assumption does not limit the generality of our model, and reflects the natural encoding in the application domain.

The proof of NP-completeness of a decision problem $\mathcal{P}$ consists of two parts. First we need to show that the problem is in the class NP, by showing that it can be solved by a non-deterministic Turing Machine in polynomial time. Technically this is equivalent to the question: Given a solution to a problem, can its correctness be verified in polynomial time? This part is straight forward to demonstrate in most cases. The second part is known as reduction. To do the reduction we chose a known NP-complete problem $Q$ and prove that $\mathcal{P}$ is at least as hard to solve as $Q$; this is done by reducing $Q$ to $\mathcal{P}$ in polynomial time, such that a solution to $\mathcal{P}$ implies that a solution to $Q$. In other words, given any instance $q$ of problem $Q$, we construct an instance $p$ of problem $\mathcal{P}$ in polynomial time and space, such that if the answer to $p$ is yes then the answer to $q$ is yes and if the answer to $p$ is no then the answer to $q$ is no. More formal descriptions about the complexity class NP and other related definitions can be found in [CLR89].

We use the following known NP-complete problem, 3-partition, to show NP-completeness of our problems.

Definition. 3-Partition: Given a multi-set $\mathcal{A}=\left\{a_{1}, a_{2}, \ldots, a_{3 w}\right\}$ and a positive integer $B$, 
where $\sum_{i=1}^{3 w} a_{i}=w B$ and $\forall i \in\{1,2,3, \ldots, 3 w\}, B / 4<a_{i}<B / 2$. For any multi-set $\mathcal{A}$ does there exists a set of $w$ partitions $A_{1}, A_{2}, \ldots, A_{w}$, such that each one has exactly 3 elements and $\sum_{a \in A_{i}} a=B, \forall i \in\{1,2, \ldots, w\}$.

Our first result shows that the SDPB problem is NP-complete. We prove that by showing in Theorem 1 that a restricted case of the problem where the number of disks is limited to two, and the buffer memory per reference string is limited to one, is NP-complete.

Lemma 3.1. $\operatorname{SDPB}(n, d, m, L, K)$ is in $N P$.

Proof. Given an I/O schedule for $n$ reference strings, it can be easily checked if the length of the combined schedule is no more than $K$, and that it is a valid schedule. To check that it is a valid schedule, we merely need to verify that for each reference string the buffer constraints are never violated; that is when the demand block is fetched there are less than $m$ other blocks from that reference string in the buffer.

Theorem 1. $S D P B(n, 2, L, 1, K)$ is NP-complete.

Proof. From Lemma 3.1 we know that the problem is in NP.

We reduce the known NP-complete problem 3-Partition to $\operatorname{SDPB}(\mathrm{n}, 2, \mathrm{~L}, 1, \mathrm{~K})$. For any 3-Partition problem, we are given $w, B$ and a multi-set $\mathcal{A}=\left\{a_{1}, a_{2}, \ldots, a_{3 w}\right\}$. We construct an instance of $\operatorname{SDPB}(\mathrm{n}, 2, \mathrm{~L}, 1, \mathrm{~K})$ with $n=3 w+1, m=1$, and $K=2 w B$. The $n$ reference strings $R_{1}, R_{2}, \cdots, R_{3 w}, R_{3 w+1}$ are defined as follows. We use the notation $\left(a_{i} \times D_{j}\right)$ to mean $a_{i}$ distinct consecutive requests for disk $D_{j}$. Also we use $X * Y$ to indicate the concatenation of substrings $X$ and $Y$, and $X^{s}$ to indicate the concatenation $s$ times of string $X$.

$$
\begin{gathered}
R_{1}=\left(a_{1} \times D_{1}\right) *\left(a_{1} \times D_{2}\right) \\
R_{2}=\left(a_{2} \times D_{1}\right) *\left(a_{2} \times D_{2}\right) \\
\vdots \\
R_{i}=\left(a_{i} \times D_{1}\right) *\left(a_{i} \times D_{2}\right) \\
\vdots \\
R_{3 w}=\left(a_{3 w} \times D_{1}\right) *\left(a_{3 w} \times D_{2}\right) \\
R_{3 w+1}=\left(\left(B \times D_{2}\right) *\left(B \times D_{1}\right)\right)^{w}
\end{gathered}
$$




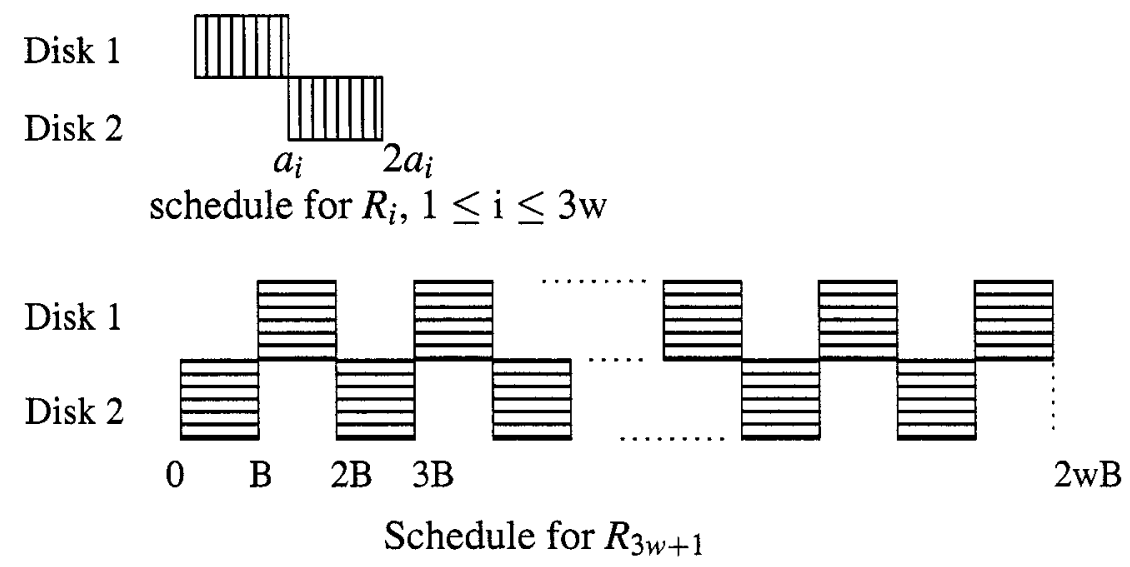

Figure 3.1 : Individual schedules for reference strings

Note that the sum of lengths of all reference strings is $(2 w B+2 w B)=4 w B$. This can be seen as follows: $R_{i}, 1 \leq i \leq 3 w$ is made up of exactly $2 a_{i}$ requests, and $\sum_{i=1}^{3 w} a_{i}=w B$, for a total length of $2 w B$. Additionally $R_{3 w+1}$ has $2 w B$ requests. Thus the size of the input to the scheduling problem is polynomially related to $\operatorname{sum} \sum_{i=1}^{3 w} a_{i}=w B$. This is important, since the 3-Partition problem is known to be NP-Complete even if the input weights are represented in unary i.e. the length of the input to 3-Partition is assumed to be of size $w B$.

First consider the following nominal schedules for individual reference strings. For all $i \in\{1,2, \cdots, 3 w\}$, the schedule for a reference string $R_{i}$ consists of $a_{i}$ block accesses from disk $D_{1}$ followed by $a_{i}$ accesses from $D_{2}$. There can be no overlap between these accesses of the two disks because there is only a buffer of size one for each reference string.

The nominal schedule for $R_{3 w+1}$ consists of $B$ block accesses from $D_{2}$ followed by $B$ block accesses from $D_{1}$, and this sequence is repeated $w$ times. Here too there are no overlaps between accesses to the two disks. These nominal schedules are shown in Figure 3.1. Note that the length of $R_{3 w+1}$ is $2 w B$, equal to the deadline $K$, of the entire schedule. Consequently, since there can be no overlap between the fetches for this reference string, it is not possible to delay any request of $R_{3 w+1}$ without exceeding the deadline.

Now we will show that a 3-partition exists if and only if there is a schedule of length $K=2 w B$. 
- If $w$ partitions $A_{1}, A_{2}, A_{3}, \cdots, A_{w}$ exist such that for each partition $A_{k}=\left\{a_{k_{1}}, a_{k_{2}}, a_{k_{3}}\right\}$, $\sum_{a_{i} \in A_{k}} a_{i}=B$, then the following $\mathrm{I} / \mathrm{O}$ schedule with length $2 w B$ is certainly possible. In Figure 3.2, the $a_{k_{1}}+a_{k_{2}}+a_{k_{3}}$ (equal to $B$ ) blocks from disk 1 of the reference strings $R_{k_{1}}, R_{k_{2}}$, and $R_{k_{3}}$ can be completely scheduled from time $(2 k-2) B$ to $(2 k-$ 1) $B$, and the same number of blocks can be scheduled on disk 2 from time $(2 k-1) B$ to $2 k B$. Ordering of individual blocks within the length $B$ time intervals does not matter as long as all of the $a_{k_{1}}+a_{k_{2}}+a_{k_{3}}$ requests to disk 1 finish before $(2 k-1) B$, and the requests for disk 2 finish before $2 k B$. Blocks of $R_{3 n+1}$ can be fetched as shown in Figure 3.2 using regions $(2 s-2) B$ to $(2 s-1) B$ on disk 2 , and $(2 s-1) B$ to $2 s B$ on disk 1 where $s \in\{1,2, \cdots, w\}$. Hence if a 3 -partition exists, so does a schedule with length $2 w B$.

- If a schedule of length $2 w B$ exists, ${ }^{*}$ then we show that set $\mathcal{A}$ can be partitioned into $w$ desired partitions $A_{1}, A_{2}, \cdots, A_{w}$. First observe that in a schedule of length $2 w B$ as shown in Figure 3.2, the shaded regions have to be occupied by requests from $R_{3 w+1}$ because all its $2 w B$ requests need to be fetched in sequential order without overlap. Hence all blocks of the remaining reference strings $R_{i}, 1 \leq i \leq 3 w$, must be fetched in the unshaded regions of Figure 3.2. Also, since there are $w B$ blocks from each disk belonging to the $R_{i}, 1 \leq i \leq 3 w$, there can be no idle period in any of the unshaded regions in a schedule of length $2 w B$.

Denote the $i^{t h}$ such unshaded region on disk 1 by $\Gamma_{i}, 0 \leq i \leq w-1 . \Gamma_{i}$ spans the interval $[2 i B,(2 i+1) B]$ and has length $B$. No $\Gamma_{i}$ can contain all the blocks (from disk 1) of more than three $R_{j}$, since $a_{j}>B / 4$, for all $1 \leq j \leq 3 w$.

As a consequence one of the following must hold. Either (a) every $\Gamma_{i}$ contains all the blocks of exactly three $R_{j}$, say $R_{i_{1}}, R_{i_{2}}$, and $R_{i_{3}}$, and only these blocks, or (b) there is some $\Gamma_{s}, 0 \leq s \leq w-1$, such that either (i) or (ii) below hold, and $s$ is the smallest such integer: (i) $\Gamma_{s}$ contains all the blocks of three reference strings $R_{s_{1}}, R_{s_{2}}, R_{s_{3}}$ and 


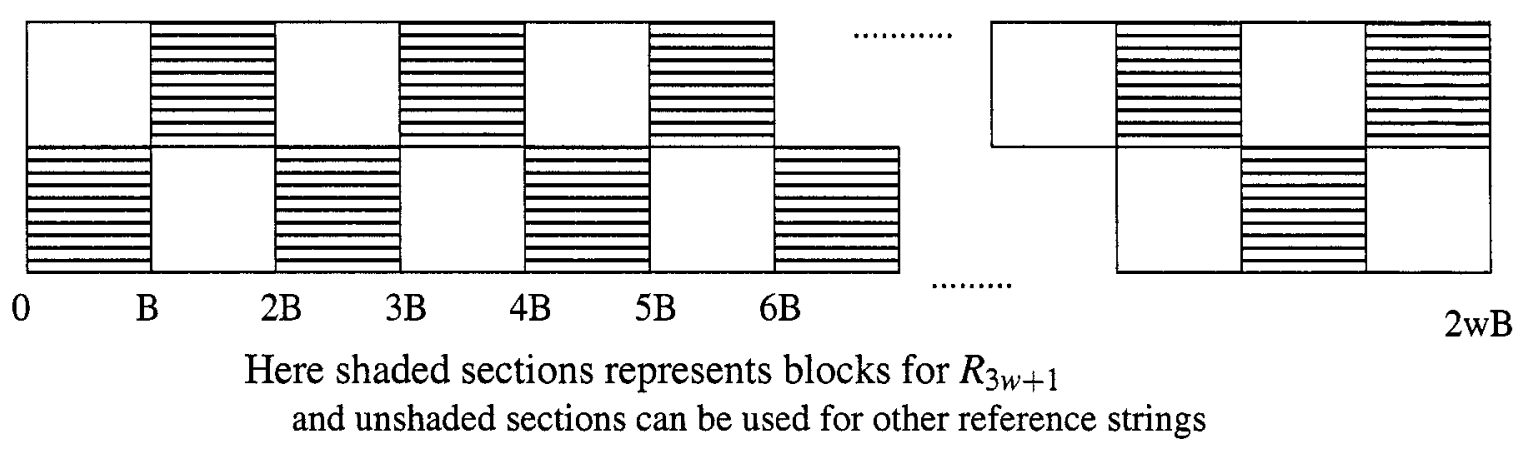

Figure 3.2 : Possible schedule with length $2 \mathrm{wB}$

at least one block from some other reference string $R_{z}$, or (ii) $\Gamma_{s}$ contains all the blocks of less than three strings.

If (a) is true, then there is a 3-Partition in which $A_{i}=\left\{a_{i_{1}}, a_{i_{2}}, a_{i_{3}}\right\}$. If case (b) is true, we will argue that there must be some idle time on $D_{2}$ between $B$ and $(2 s+1) B$, contradicting the existence of a schedule of length $2 \mathrm{wB}$. Now blocks on $\mathrm{D}_{2}$ can only be scheduled when blocks of that reference string on $D_{1}$ have all been scheduled. In case (b), up to time $2 s B$, there were $s B$ blocks that were ready to schedule on $D_{2}$, while between time $(2 s+1) B$ and $(2 s+2) B$ there will be less than $B$ new blocks to schedule on $D_{2}$. This is because in case (i), $a_{s_{1}}+a_{s_{2}}+a_{s_{3}}<B$, and in case (ii) the sum of less than three $a_{j}$ is less than $B$.

Hence, there will be an idle period on $D_{2}$ sometime during the unshaded interval between $(2 s+1) B$ to $(2 s+2) B$, contradicting the existence of a schedule of length $2 w B$.

Now we will show that the SDSB problem is NP-complete. We show that by proving a restricted case with 2 disks to be NP-complete.

Lemma 3.2. $\operatorname{SDSB}(n, d, M, L, K)$ is in $N P$. 
Proof. The proof is quite similar to the proof of Lemma 3.1. Here again given an $\mathrm{I} / \mathrm{O}$ schedule for multiple reference strings, it can be easily checked if it a valid schedule and has length no more than $K$. To check the validity of the schedule, we just need to verify that total number of prefetched blocks is always less than $M$.

Theorem 2. $S D S B(n, 2, M, L, K)$ is NP-complete.

Proof. From Lemma 3.2 we know that the problem is in NP.

We reduce the known NP-complete problem 3-Partition to SDSB(n,2,M,L,K). For any 3-Partition problem, we are given $w, B$ and a multi-set $\mathcal{A}=\left\{a_{1}, a_{2}, \ldots, a_{3 w}\right\}$. We construct an instance of $\operatorname{SDSB}(\mathrm{n}, 2, \mathrm{M}, \mathrm{L}, \mathrm{K})$ with $n=3 w+1, M=c B$ for some constant $c$, and $K=$ $2 w \alpha-M+1$, where $\alpha=B+M-1$. The reference strings $R_{1}, R_{2}, \cdots, R_{3 w}, R_{3 w+1}$ are defined as follows. Here again notation $\left(a_{i} \times D_{j}\right)$ means $a_{i}$ distinct consecutive requests for disk $D_{j}, X * Y$ denotes concatenation of substrings $X$ and $Y$, and $X^{s}$ indicates $s$ consecutive occurrences of $X$.

$$
\begin{gathered}
R_{1}=\left(a_{1} \times D_{1}\right) *\left(a_{1} \times D_{2}\right) \\
R_{2}=\left(a_{2} \times D_{1}\right) *\left(a_{2} \times D_{2}\right) \\
\vdots \\
R_{i}=\left(a_{i} \times D_{1}\right) *\left(a_{i} \times D_{2}\right) \\
\vdots \\
R_{3 w}=\left(a_{3 w} \times D_{1}\right) *\left(a_{3 w} \times D_{2}\right) \\
R_{3 n+1}=\left(\alpha \times D_{2}\right) *\left[\left((\alpha+M-1) \times D_{1}\right) *\left((\alpha+M-1) \times D_{2}\right)\right]^{(w-1)} *\left(\alpha \times D_{1}\right)
\end{gathered}
$$

Note that the sum of lengths of all reference strings is $(2 w B+2 \alpha+(w-1)(\alpha+M-$ $1)=4 w B+(4 w-2)(M-1)$ which is clearly polynomially bounded by the $\operatorname{sum} \sum_{i=1}^{3 w} a_{i}=$ $w B$ when $M=\Theta(B)$.

Individual schedules for each reference string would be as follows: For all $i \in\{1,2, \cdots$ $, 3 w\}$, the schedule $R_{i}$ consists of $a_{i}$ fetches from $D_{1}$ followed by $a_{i}$ fetches from $D_{2}$ with some overlap possible between the fetches from $D_{1}$ and $D_{2}$. The amount of overlap can be no more than $M-1$. $R_{3 w+1}$ consists of $\alpha$ blocks from $D_{2}$ followed by a repeating pattern 


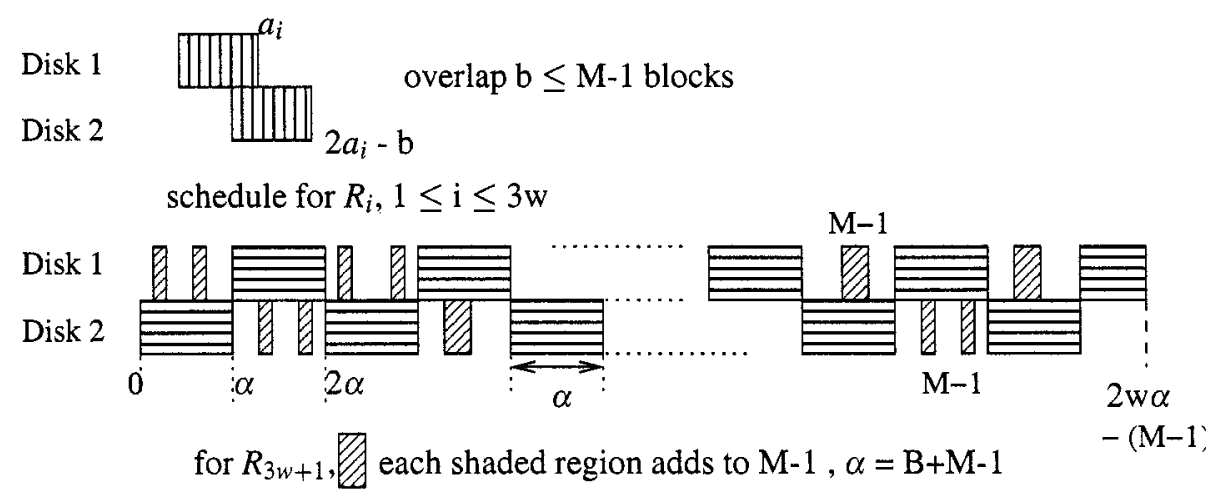

Figure 3.3 : Individual schedules for shared memory case

consisting of $\alpha+M-1$ blocks from $D_{1}$ followed by $\alpha+M-1$ blocks from $D_{2}$; this pattern is repeated $w-1$ times, and is finally followed by $\alpha$ blocks from $D_{1}$. Individual schedules for $R_{i}$ are shown in Figure 3.3. In order for $R_{3 w+1}$ to complete within the schedule length $K$, the following is necessary: in the interval $(k-1) \alpha$ to $k \alpha, k=\{1,2, \cdots, w-1\}, \alpha$ blocks of $R_{3 w+1}$ must be fetched from $D_{2}$ if $k$ is odd, and from $D_{1}$ if $k$ is even. In order to meet this schedule there must be an overlap of $M-1$ fetches of $R_{3 w+1}$ in every interval $[(k-1) \alpha, k \alpha]$. This implies that there are no free buffers available for prefetching blocks of $R_{i}, 1 \leq i \leq 3 w$, which must be fetched one block at a time.

Now we will show that if there exists a 3-Partition then a schedule of length $K=2 w \alpha-$ $(M-1)$ is possible, and if a schedule of length $K$ is possible, then a 3-Partition exists for set $\mathcal{A}$.

- If a partition of $\mathcal{A}$ into $w$ subsets $\left\{A_{1}, A_{2}, A_{3}, \cdots, A_{w}\right\}$ exists, such that $A_{k}=\left\{A_{k_{1}}, A_{k_{2}}\right.$ ,$\left.A_{k_{3}}\right\}, \sum_{k_{i}} a_{k_{i}}=B$, then the following schedule of length $(2 w-1) \alpha+B$ is certainly possible.

In Figure 3.4, for each unshaded region $2 k \alpha$ to $(2 k+1) \alpha$, requests corresponding to $A_{k}$, in particular reference strings $R_{k_{1}}, R_{k_{2}}$ and $R_{k_{3}}$, are scheduled on $D_{1}$. The corresponding requests from $D_{2}$ are scheduled from time $(2 k+1) \alpha$ to $(2 k+2) \alpha$. Within this unshaded region of length $\alpha$ the $B$ blocks from $R_{k_{i}}$ and the $M-1$ prefetched 


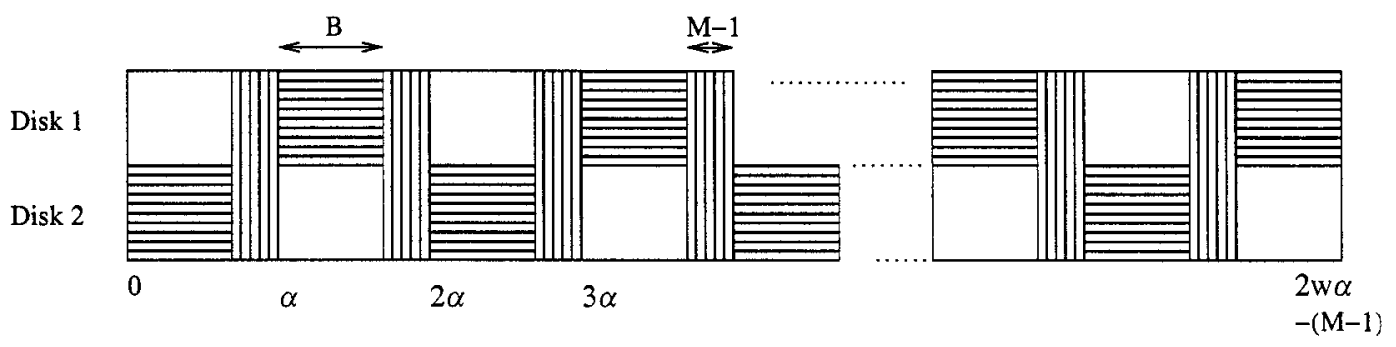

Figure 3.4 : Possible schedule with length $2 \mathrm{w} \alpha-(\mathrm{M}-1), \alpha=\mathrm{B}+\mathrm{M}-1$

blocks from $R_{3 w+1}$ are fetched; their relative ordering within the region is unimportant. Similarly the unshaded region on $D_{2}$ is used to schedule the $B$ blocks on $D_{2}$ from $R_{k_{i}}$ and the $M-1$ prefetched blocks from $R_{3 w+1}$, as shown in figure 3.4. Hence if a 3-Partition exists, so does a schedule with length $K$.

- If a schedule of length $(2 w-1) \alpha+B$ exists, then we show that set $\mathcal{A}$ can be partitioned into $w$ desired subsets $A_{1}, A_{2}, \cdots, A_{w}$. First observe that in a valid schedule, $R_{3 w+1}$ in Figure 3.3 needs all the shaded regions in Figure 3.4, and $M-1$ of each unshaded intervals for prefetching. Consequently the $R_{i}, 1 \leq I \leq 3 w$ must be scheduled within $B$ length intervals in the unshaded regions. The conditions are exactly the same as in Theorem 1, where the $R_{i}$ needed to be scheduled in length $B$ intervals on each disk. Using identical reasoning, we can conclude that the scheduling of the $R_{i}$ into the unshaded intervals induces a 3-Partition of $\mathcal{A}$.

\subsection{Optimal Algorithm for restricted case}

In the previous section we showed that the general problem of scheduling multiple reference strings with minimum I/O's is NP-complete. In this section we look at the complexity of the optimal scheduling problem with some restrictions on memory buffer size. We present a dynamic programming approach toward deterministic optimal algorithm for such restricted cases. Our approach works in polynomial time for a fixed number of strings, 
within the bound $\mathrm{O}\left(L^{N}\right)$, where $\mathrm{L}$ is the maximum length of a string and $N$ is the total number of strings that is fixed.

Theorem 3. Given $N$ reference strings with maximum length $L$ per string, a set of $d$ disks that are shared between all of them and a buffer of size 1 per string, the optimal schedule with minimum number of $I / O$ 's can be constructed in $O\left(L^{N}\right)$ time and $O\left(L^{N-1}\right)$ space.

Algorithm: We first explain the algorithm for a simple case when $N=2, D \geq 2$ and is generalized later on for any N. Let us take 2 strings of length $\mathrm{L}$ each. Since we have a buffer of size 1 block per string, we always have to fetch the demand block and hence no prefetching is possible. For each $\mathrm{I} / \mathrm{O}$ we look at only one block from each string. Hence each $\mathrm{I} / \mathrm{O}$ can have one of the following 3 cases:

1. requests from both the strings belong to different disks and so we can schedule both of them together.

2. requests from both the strings belong to a single disk and we fetch a block that belongs to string 1 .

3. requests from both the strings belong to a single disk and we fetch a block that belongs to string 2 .

Thus at each step we decrease the required number of blocks for at least one string . Let $(\mathrm{n}, \mathrm{m})$ denotes the state in which we have fetched $n$ blocks from string 1 and $\mathrm{m}$ blocks from string 2 . Ultimately, we want to go from state $(0,0)$ to $(L, L)$ by taking some path in a two-dimensional space as shown in the Figure 3.5. Although the total number of possible paths is exponential $O\left(2^{L}\right)$, we can find a shortest path in $O\left(L^{2}\right)$ time using dynamic programming.

At each step, the algorithm maintains a set $\mathbf{S}$ of all possible states of the algorithm and use it to determine the next set. In Figure 3.5 XY and $\mathrm{CD}$ denotes possible sets of state during the execution of the algorithm. At each step, we discard a pair $(\mathrm{x}, \mathrm{y})$ if it is strictly smaller than any other pair in the set. A pair $\left(x_{1}, y_{1}\right)$ is strictly smaller than $\left(x_{2}, y_{2}\right)$ iff 


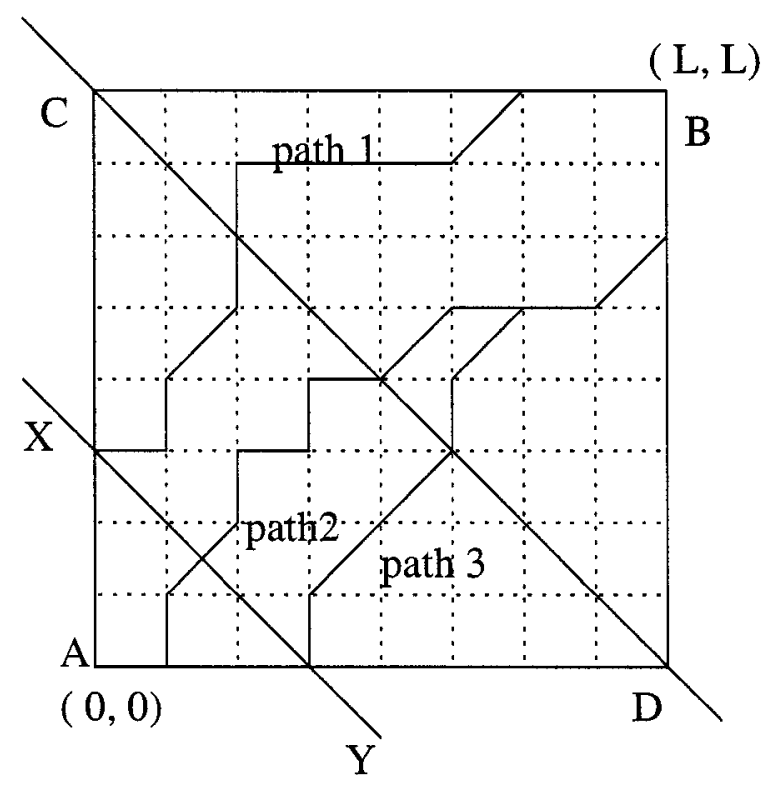

Figure 3.5 : Possible paths with two strings

$\left(x_{1} \leq y_{1}\right.$ and $\left.x_{2} \leq y_{2}\right)$. For example, if the set contains $\{(2,3),(1,4),(0,5),(3,2),(2,2)$, $(1,3)\}$, we will discard $(2,2)$ and $(1,3)$ because they are strictly smaller than $(3,2)$ and $(1,4)$ respectively. Hence the maximum size of set $\mathrm{S}$ can be $O(L)$ as shown by the diagonal $\mathrm{CD}$. At each state $(\mathrm{x}, \mathrm{y})$, we only need to remember the minimum cost path leading to $(\mathrm{x}, \mathrm{y})$, where cost is measured as number of edges taken to reach $(\mathrm{x}, \mathrm{y})$. Also from each state we only have $\left(2^{N}-1\right)$ options to consider to calculate next set of states. Hence we just need $3 \times O(L)$ computation at each step. Total number of such steps is $O(L)$ as longest path can be of length $2 \times L$. So complexity of algorithm can be $O\left(L^{2}\right)$ in worst case. This bound can be achieved because a lot of different paths converge at a point (for example paths 2 , 3 converge and have a common suffix of length 4) to give us an opportunity to explore a search space ${ }^{\dagger}$ in polynomial space and time using dynamic programming. This feature has been observed for many other problems as well. Now for more general values of N, D we

\footnotetext{
${ }^{\dagger}$ It consists of all the possible paths that an algorithm might take in going from initial to final state, which is $(0,0)$ to $(L, L)$ in this case. Clearly total number of possible paths is exponential in terms of input size.
} 
have the following cases for the search space:

1. $\mathbf{N} \leq \mathbf{D}$ : Next block required by a string can belong to any of the $D$ disks. So we have an option to fetch or not to fetch a block from any of the strings. We have to fetch at most one block from any disk in one $\mathrm{I} / \mathrm{O}$ if all the strings need to access same disk in current $\mathrm{I} / \mathrm{O}$. This gives us $2^{N}-1$ options at each step. Total number of steps is bounded by $L \times N$ where $\mathrm{L}$ is maximum string length. The number of total possible ways is $O\left(\left(2^{N}-1\right)^{O(L N)}\right)$.

2. $\mathbf{N}>\mathbf{D}$ : Here only $\mathrm{D}$ out of $\mathrm{N}$ strings can be chosen for a single $\mathrm{I} / \mathrm{O}$. So we have $N_{C_{1}}+$ $N_{C_{2}}+\cdots+N_{C_{D}}=O\left(N^{D}\right)$ options at each step. Total number of steps is bounded by $L \times N$ where $\mathrm{L}$ is maximum string length. The total number of possible ways is $O\left(\left(N^{D}\right)^{O(L N)}\right)$. Hence the search space is exponential.

Although search space is exponential in terms of input in all cases, we can use dynamic programming and keep the set of all possible current states of the strings. For any $\mathrm{N} \mathrm{di-}$ mensional space, the maximum space required is $O\left(L^{N-1}\right)$. Also from each state there are $\left(2^{N}-1\right)$ ways to go to the next state. Thus the computation required at each step to compute the next set of states is $\left(2^{N}-1\right) \times O\left(L^{N-1}\right)$ and total number of steps is bounded by LN. So the time complexity of algorithm is $O\left(\left(2^{N}-1\right) N L^{N}\right)$ and space requirement is $O\left(L^{N-1}\right)$.As shown in the figure 3.6 when $\mathrm{N}=3$, the maximum size of state space at an instant is a plane, which is $O\left(L^{2}\right)$. The input of the problem is measured as the sum of lengths of all reference strings that is $\mathrm{O}(\mathrm{LN})$ and for a fixed $\mathrm{N}$ the running time of our algorithm is $\mathrm{O}\left(L^{N}\right)$ and space required is $\mathrm{O}\left(L^{N-1}\right)$.

\subsection{Optimal Static Memory Allocation for the PDSB Model}

In this section we consider the model where each reference string has its own set of disks disjoint from the others. There is a fixed amount of buffer memory that will be statically partitioned among the reference strings, so that the schedule length of the concurrently accessed reference strings is minimized. In this model, the length of the overall schedule 


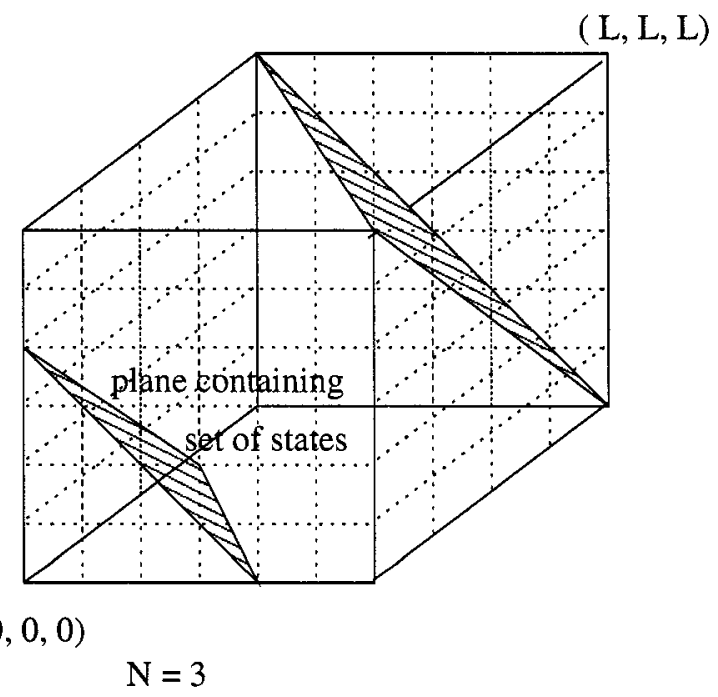

Figure 3.6 : Possible paths and space requirement with three strings

is determined by the longest individual schedule since these can all operate concurrently without conflict. The length of each individual schedule in turn depends on the amount of buffer memory allocated to it. We design a buffer allocation algorithm so as to minimize the maximum individual schedule length.

Given $n$ reference strings each of length $L$ and a buffer memory of size $M$, a straightforward algorithm can consider all n-partitions of $\mathrm{M}$ (and choose the partition that minimizes the maximum length schedule. This assumes that the existence of an algorithm that computes the number of $\mathrm{I} / \mathrm{O}$ 's required for a single string given a certain size the memory buffer. The algorithm provided in $[\mathrm{KV}]$ to find the optimal schedule for a single reference string can be used. However, since there are $\Theta\left(M^{n}\right)$ such partitions when ordering of partitions is important, this straightforward algorithm is infeasible in practice.

We present an algorithm to deterministically assigns memory blocks to all strings, in time $O\left(L n(\log M)^{\log n}\right)$, so as to minimize the maximum number of $\mathrm{I} / \mathrm{Os}$ of any string. First, we present an efficient algorithm for the case $n=2$ and then show how it can be generalized for arbitrary number $n$ of reference strings.. 


\section{for all $m$ from 1 to $M-1$}

Assign $m$ memory buffers to string 1 and $(M-m)$ buffers to string 2

Let $L_{j}^{m}$ denote the length of the minimum schedule for string $j$ using buffer of size $m$. calculate $L_{1}^{m}$ and $L_{2}^{M-m}$; length $=\max \left(L_{1}^{m}, L_{2}^{M-m}\right)$;

if (length $<\min$ ) minbuf $=\mathrm{m} ; \min =$ length;

return min, minbuf;

Figure 3.7 : Simple static allocation algorithm for two strings

The straightforward algorithm for $n=2$ requires $O(L M)$ time, and is shown in Figure 3.7. It computes the schedule length for each reference string for all allocations of the buffer memory between 1 and $M$, and then finds the allocation $m$, such that allocating $m$ blocks of buffer to reference string 1 and $M-m$ blocks to reference string 2, minimizes the maximum individual schedule.

A more efficient algorithm that requires only $O(L \log M)$ time instead of $O(L M)$ is shown in Figure 3.8. The main idea is as follows: we look at the schedule lengths for string 1 with buffer size $m$ and string 2 with buffer size of $M-m$. If the schedule length of reference string 1 is the larger we should allocate more memory to it and less to reference string 2 , or vice versa if reference string 2 has the longer schedule. By reallocating the excess buffer between the two strings, using a binary-search like pattern, we can converge on the optimal in a logarithmic number of probes.

For $n$ reference strings, we use a recursive version of the above method. Divide the strings into two parts of size $n / 2$ strings each. Assign $m_{1}$ buffer blocks to the first partition and $m_{2}$ buffer blocks to the other partition. Initially, $m_{1}=m_{2}=M / 2$. Recursively, find the best schedule length for each partition using $m_{1}$ and $m_{2}$ buffers respectively. If the first partition has the longer schedule length, then move buffers from partition 2 to partition 1 , or vice versa as necessary. Continue the process till one converges on the best allocation. 


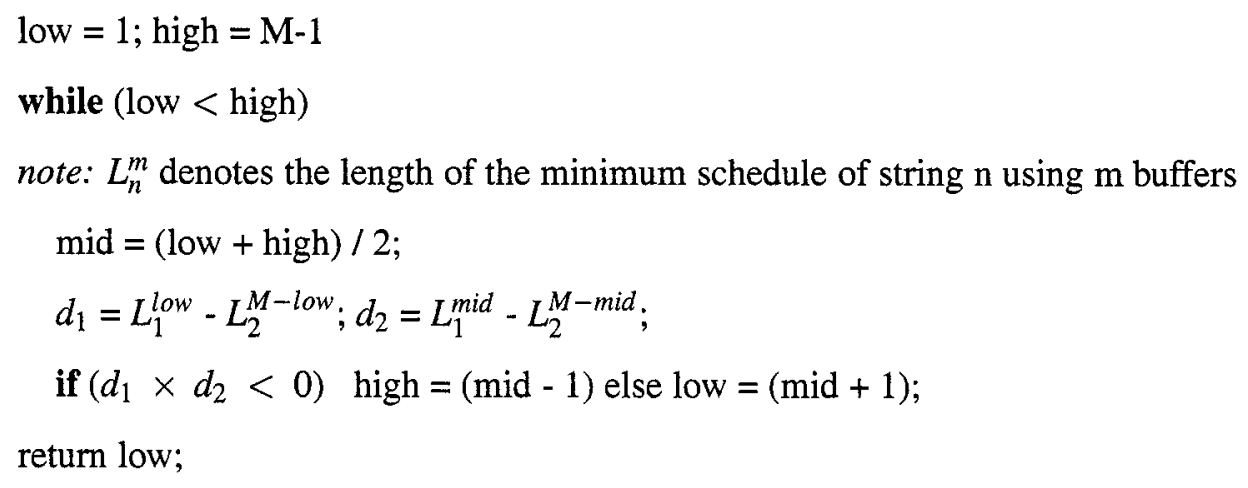

Figure 3.8: $O(L \log M)$ algorithm for two strings

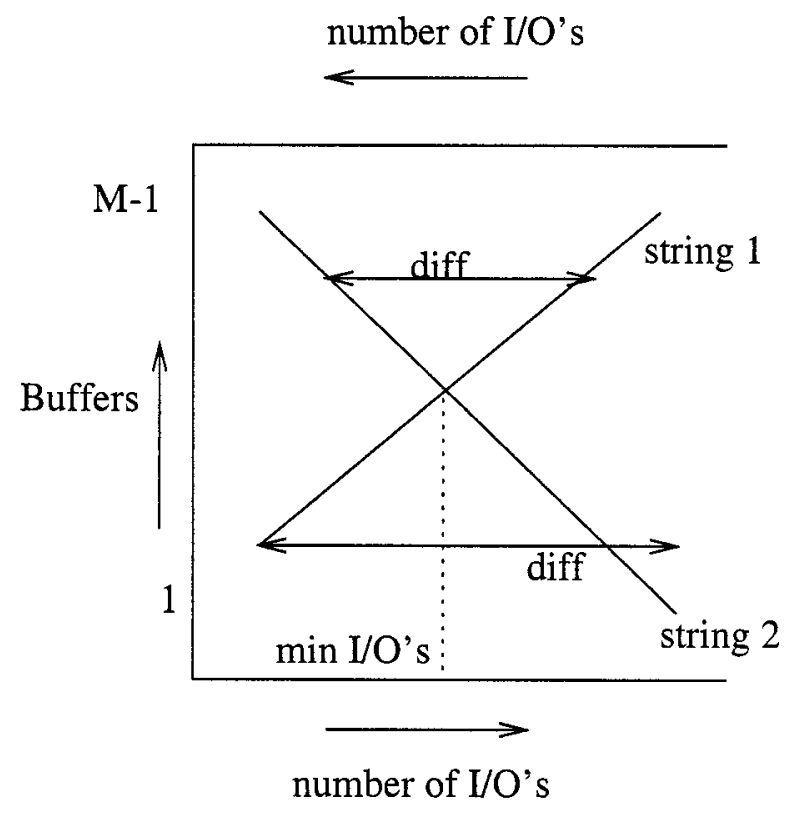

Arrows shows direction of increase of values initial and final values can be different on two $\mathrm{x}$ axis

Figure 3.9 : Convergence in $\log (\mathrm{M})$ steps 
We will require to make $\Theta(\log M)$ probes to each of the subproblems to find the best allocation.

Formally this recursion can be represented as:

$$
\begin{gathered}
T(n, M) \leq c(\log M)(2 T(n / 2, M)) \\
T(1, M) \leq c_{1} L
\end{gathered}
$$

Solving the recurrence, we get:

$$
T(n, M) \leq \log ^{k} M 2^{k} T\left(N / 2^{k}, M\right) \leq c N L(\log \log n) M
$$

Hence for $n$ strings of maximum length $L$, the upper bound will be $O\left(n L\left(\log ^{\log n} M\right)\right)$.

\subsection{Approximate Algorithms}

Since the problem of optimally scheduling multiple read once reference string with shared disks and distributed or shared memory buffers is NP-complete, we looked at some heuristics to use in practice. A brute-force method finds an optimal algorithm for each individual strings, and concatenates them serially. This will result in a schedule of length $=O\left(N L_{\max }\right)$ where $L_{\max }$ is the length of the longest individual schedule. There are input strings for which this is the best possible schedule. Although we were unable to find a heuristic with provably better worst-case performance than this brute-force method, we believe some of the following approaches may provide better typical or average case performance.

\subsubsection{Static Priority Assignment}

This heuristic assigns a static priority to all the reference strings and orders them according to the priority value. Whenever there is a conflict between two strings for the same disk, the string with highest priority is serviced. The optimal algorithm might give different priorities to different strings at different times to resolve conflicts, so this strategy cannot be optimal. One refinement we investigated was to calculate the schedule for all possible $n$ ! priority assignments, and take the minimum. Although in our (small) experimental 


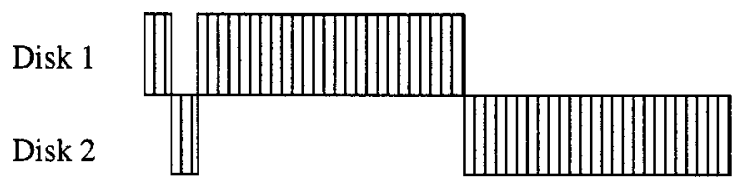

Schedule for reference string 1

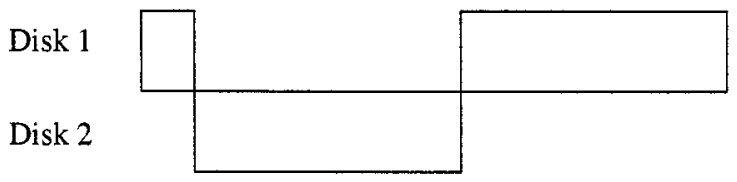

Schedule for reference string 2

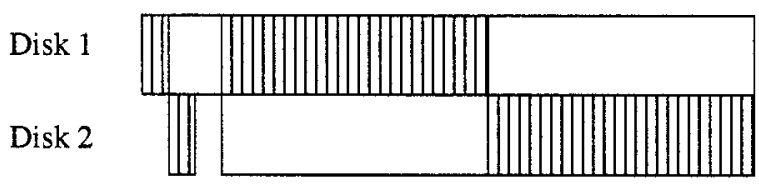

Optimal Schedule for both strings

Figure 3.10 : Bad case for Static priority Assignment Heuristic

sample this performed much better than the worst-case priority choice, it is still possible to obtain a schedule that is as bad as $\Theta(n)$ times the optimal. The following example shows a pathological case for this heuristic. Consider the two reference strings in Figure 3.10, each of length $L$, and a partitioned buffer of size $m=1$ per string. The optimal schedule of length $L+2$ can be constructed as shown in Figure 3.10, but this heuristic gives a schedule of minimum length $2 L-2$ as shown in figure 3.11 .

\subsubsection{Merge and Schedule}

This heuristic merges all strings by picking one block from each in a round robin fashion. It then schedules that string as if it was a single string by using the optimal algorithm to schedule a single string. Although we simulated this heuristic and tried it on random input strings, it is hard to say how well it performs in absence of any benchmarks. We compared it with the optimal schedule generated in the restricted case of one memory buffer per 


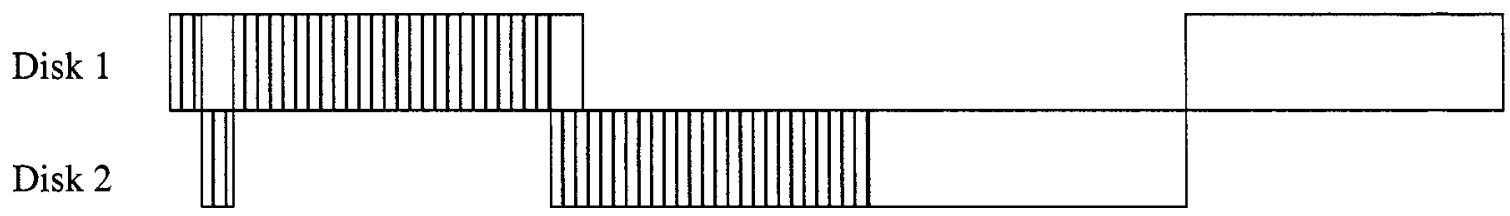

Hueristics's schedule when reference string 1 has higher priority

Disk 1

Disk 2

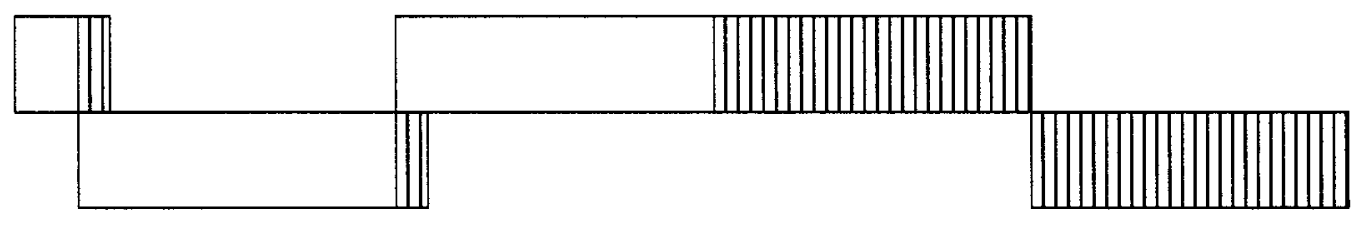

Hueristics's schedule when reference string 2 has higher priority

Figure 3.11 : Schedules created by Static Priority Assignment Heuristic

reference string and it performs well in terms of obtaining schedules comparable with the schedules generated with our optimal algorithm.

\subsection{Related work}

The foundation of the complexity class NP was laid by Stephen Cook in his seminal paper "The Complexity of Theorem-Proving Procedures" in 1971 [Coo71]. He emphasized the concept of polynomial time reducibility and proved the problem of Satisfiability to be NP-complete. Since then hundreds and thousands of problems have been added to this set by using the concept of reduction. Garey and Johnson [GJ79] published a widely-used compendium of NP-complete problems in 1979. A lot of scheduling problems such as multiprocessor scheduling and shop scheduling have been shown to be NP-complete. Our proof of NP-completeness is inspired by the proofs of Flow-Shop [GS78] and Job-Shop [GJS76] scheduling problems. Look at [GJ79] for more detailed definitions of these problems, and the cases for which these problems can be solved in polynomial time. Jain et al. [JSWB97] have presented some heuristics for efficient scheduling in parallel $\mathrm{I} / \mathrm{O}$, but they consider a slightly different problem of assigning disks to processors using edge coloring of a graph. 
In their schedule only one disk is assigned to one processor at each step, whereas in our case a string can access multiple disks in single I/O. In any case, that algorithm also does not guarantee an optimal schedule.

The problem of scheduling a single reference string on multiple disks has been studied extensively in literature $\left[\mathrm{KCF}^{+} 96, \mathrm{KV} 99 \mathrm{a}, \mathrm{AGL} 00, \mathrm{BKVV} 00, \mathrm{KV} 01, \mathrm{KV}\right]$ and polynomialtime algorithms for some of its variants have been discovered. We are not aware of any formal work that addresses the problem of scheduling multiple independent workloads on a parallel I/O system. 


\section{Chapter 4}

\section{Scheduling with Fairness/Weighted-QoS}

In Chapter 3, we showed that the problem of maximizing the disk bandwidth utilization while scheduling a set of $\mathrm{n}, n>1$, reference strings is NP-complete. We also looked at some heuristics that perform well with certain inputs but none of them has a worst case bound less than that obtained by scheduling all the $n$ reference strings sequentially. Besides being computationally intractable and requiring a priori knowledge of the entire set of reference sequences, such a minimal-length schedule may still be unattractive from the viewpoint of fairness in the allocation of the disk bandwidth to individual applications.

The problem of fair or weighted assignment of storage bandwidth become much more significant for large storage systems. Some of the reasons for this are:(1) the transfer rates are quite high (2) workloads served might belong to different organizations with different bandwidth requirement (3) workloads are unpredictable in nature and might affect each other's performance (4) workloads change over time and its difficult to provision for worst case scenarios. We investigate the problem of fairness and weighted allocation of disk blocks among workloads.

In this chapter we present a model for fair disk bandwidth allocation among concurrent tasks in a parallel I/O system, and present efficient scheduling algorithms for implementing several allocation policies. By necessity, our model is an idealized abstraction of a complex parallel I/O system where mechanical disk delays and interconnect scheduling issues need to be considered. Many of these factors can be captured by a more detailed model; this work will concentrate on the higher-level scheduling issues. 


\subsection{Overview}

A parallel I/O system consists of a set of $d$ independent disks $\mathcal{D}=\left\{D_{1}, D_{2}, \cdots, D_{d}\right\}$. Data is stored on the disks in units of blocks; a block is the unit of access from a disk. In each parallel I/O operation a set of up to $d$ blocks, one from each disk, can be accessed. The blocks fetched from the disks may be buffered in internal memory buffer until they are required. The presence of the buffer allows applications to prefetch blocks before they are required and reduce the overall $\mathrm{I} / \mathrm{O}$ latency.

A set of $n$ independent applications or tasks is assumed to be concurrently accessing the $\mathrm{I} / \mathrm{O}$ system. Each task is abstracted by a reference string $R_{i}, 1 \leq i \leq n ; R_{i}$ is the ordered sequence of blocks that is required by that application. A reference string can be a very general representation for a sequence of disk requests that belongs to a customer, set of applications, set of users etc. For each reference string the system has a lookahead of $L-1$ blocks, $L \geq 1$, beyond the current request from that string. This lookahead enables the application to prefetch blocks that will be accessed in the near future and hold them in the buffer until required.

An $I / O$ schedule consists of a sequence of parallel $\mathrm{I} / \mathrm{O}$ steps: in each step at most one block from each of the $d$ disks is accessed. An V/O schedule is said to be work conserving if every I/O step employs maximal parallelism: that is, a disk is busy unless there is no request for that disk or there is not enough buffer space to hold the accessed block. In this chapter we will assume that the buffer is large enough to hold the lookahead $L$ of all the strings: hence in a work-conserving schedule only disks for which there are no requests at that step will be idle. Under this assumption of limited lookahead, it has been shown that a work conserving schedule minimizes the number of $\mathrm{I} / \mathrm{O}$ steps required for any single reference string in isolation [BKVV00].

A parallel I/O step will be represented by a fetch vector, $\mathrm{F}=\left[b_{1}, b_{2}, \cdots, b_{n}\right]$, where $b_{i}$ is the number of blocks from reference string $R_{i}$ that are fetched in that $\mathrm{I} / \mathrm{O}$ step. Note that since at most $d$ blocks can be fetched in any parallel I/O step, $\sum_{1 \leq i \leq n} b_{i} \leq d$. The cumulative fetch vector, $\mathrm{CF}=\left[B_{1}, B_{2}, \cdots, B_{n}\right]$, where $B_{i}$ is the total number of blocks from 
reference string $R_{i}$ fetched so far. Note that CF can be obtained by the component-wise addition of the fetch vectors for each of the preceding I/O steps. We refer to the sum of the components of any vector as the weight of the vector.

We will be interested in constructing work conserving schedules since such schedules will maximize the disk utilization at every I/O step. In addition, we would like the schedule at each step to be fair: by fairness we intuitively mean that we try to fetch as evenly as possible for all the reference strings. Formally, we say that the schedule at a step is fair if the fetch vector $\mathrm{F}$ at that step is lexicographically smallest among all $n$-vectors (i.e. vectors with $n$ components) with the same weight. We formally state the definition of lexicographic minimum below:

Consider two vectors $\mathrm{F}=\left[f_{1}, f_{2}, \cdots f_{n}\right]$ and $\mathrm{G}=\left[g_{1}, g_{2}, \cdots, g_{n}\right]$, such that $\sum_{i} f_{i}=\sum_{i} g_{i}$, and that $f_{i} \geq f_{i+1}$ and $g_{i} \geq g_{i+1}$, for all $1 \leq i \leq n-1$. Then $\mathrm{F}$ is lexicographically smaller than $\mathrm{G}$ if and only if there is some index $k, k \geq 1$, such that $f_{i}=g_{i}, 1 \leq i \leq k-1$ and $f_{k}<g_{k}$. For example consider the following 3 -component vectors with weight $7:[2,0,5],[1,3,3]$, $[3,2,2],[1,6,0],[2,4,1],[1,15]$. When arranged in non increasing order of their component values, these are the distinct vectors $[5,2,0],[3,3,1],[3,2,2],[6,1,0],[4,2,1],[5,1,1]$. The lexicographically smallest of these vectors is $[3,2,2]$ corresponding to the most balanced distribution of the component values.

The theory of partitions allows one to calculate the number of partitions in an $n$-vector of weight $w$. When the order of the components is important, this is exponential in the number of components $\left(\Theta\left(w^{n}\right)\right)$. We define three different policies for scheduling.

1. Locally Fair Allocation: Achieve a fair schedule at each $\mathrm{V} / \mathrm{O}$ step that is work conserving.

2. Globally Fair Allocation: Achieve a fair work-conserving schedule based on the cumulative numbers of blocks accessed by each string.

3. Proportional Allocation: Each reference string is assigned a weight, and the cumulative number of blocks after any step should be in proportion to their weights. 


\subsubsection{Why Lexicographic Minimum?}

A fair allocation of a resource among users requires the following:

1. every user gets an equal share

2. share $s_{1}$ of a user cannot be increased without decreasing the share $s_{2}$ of another user, where $s_{2} \leq s_{1}$ i.e. a user cannot be made better off without hurting someone with equal or less share.

This criterion is also defined as MaxMin fairness in literature [Jaf81,LS86]. A lot of work exists on MaxMin fairness in wireless and wireline networks [Hah91, TS02]. We provide more references in the related work section of this chapter. Intuitively it means to maximize the allocation to the user with minimum share. Also if a user cannot utilize its share, the remaining share is distributed equally among other users. In our case disk bandwidth allocation is MaxMin fair if it is not possible to increase the number of disks assigned to a string without hurting another string with equal or less number of disks. Another way to present the MaxMin fairness criterion is lexicographic optimality. Informally speaking a lexicographic optimal rate vector maximizes the minimum component, then the second minimum and so on in a feasible set of rate vectors. In our case optimality is defined as obtaining a minimum vector because the entries are sorted in the decreasing order. Using lexicographic minimality we can directly compare two allocation vectors for fairness as described in the previous section. Hence we use lexicographic minimality as our criterion for fair allocation. We present algorithms to achieve lexicographic optimal vectors for disk allocation among reference strings.

\subsubsection{Mapping Disk Scheduling to a Flow problem}

At any time, there are a set of candidate blocks from each reference string that can be fetched in this I/O step. These are the unfetched blocks in the lookahead for that string. The system is modeled by an augmented bipartite resource graph $G=\left(V \bigcup\{s, t\}, E \bigcup E_{t} \cup E_{s}\right)$ defined as follows: 
- $V=\left\{D_{1}, D_{2}, \cdots, D_{d}\right\} \bigcup\left\{R_{1}, R_{2}, \cdots, R_{n}\right\}$ is a set of $n+d$ nodes, one for each reference string and disk in the system.

- $\mathrm{E}$ is the set of directed edges between nodes representing reference strings and nodes representing disks: there is an edge $\left(R_{i}, D_{j}\right)$ whenever there is a request to disk $D_{j}$ in the current lookahead window of reference string $R_{i}$.

- Distinguished vertices $s$ and $t$ will serve as the source and sink of paths through the graph.

- $E_{t}=\left\{\left(D_{j}, t\right), 1 \leq j \leq d\right\}$, is the set of directed edges from each disk node to $t$.

- $E_{s} \subseteq\left\{\left(s, R_{i}\right), 1 \leq i \leq n\right\}$, is a subset of the directed edges from $s$ to string nodes $R_{i}$; this subset changes dynamically as the algorithm proceeds.

An example construction of the resource graph with 3 reference strings, 5 disks and a window size of 5 is shown in figure 4.1. We will show that the scheduling problems defined above can be mapped to finding a set of paths in a dynamically evolving resource graph. Initially the resource graph consists of $G$ defined above with $E_{s}$ being empty. Our algorithms will maintain a priority vector, $\mathcal{P}=\left[p_{1}, p_{2}, \cdots, p_{n}\right]: p_{i}$ is the priority for string node $R_{i}$. At each step the node with highest-priority is selected, and an edge from $s$ to this node is added to $E_{s}$. The algorithm then attempts to find a path in the current resource graph from $s$ to $t$ using the edge between $s$ and the selected node.

If a path cannot be found through the currently selected node $R_{i}$, then the node is marked as saturated, and the algorithm will adjust its priority so that $R_{i}$ will not be selected again. A saturated node means that it is not possible to increase the total number of scheduled disks by making a further assignment to this reference string (i.e. the weight of the fetch vector will not increase). An assignment to a saturated node will come at the expense of reducing the assignment to one of the previously scheduled nodes. The choice of priorities is such that this reallocation is undesirable.

If the search for a path is successful this means that the weight of the fetch vector can be increased by assigning an additional disk to $i^{\text {th }}$ string. The previous assignment of disks 
String 1: $\mathrm{D}_{2} \mathrm{D}_{3} \mathrm{D}_{4} \mathrm{D}_{4} \mathrm{D}_{4} \mathrm{D}_{1} \mathrm{D}_{1} \mathrm{D}_{5} \mathrm{D}_{3} \mathrm{D}_{3} \mathrm{D}_{3} \mathrm{D}_{5} \mathrm{D}_{5}$

String 2: $\mathrm{D}_{2} \mathrm{D}_{5} \mathrm{D}_{5} \mathrm{D}_{5} \mathrm{D}_{2} \mathrm{D}_{2} \mathrm{D}_{2} \mathrm{D}_{3} \mathrm{D}_{1} \mathrm{D}_{1} \mathrm{D}_{4} \mathrm{D}_{4} \mathrm{D}_{4}$

String 3: $\mathrm{D}_{4} \mathrm{D}_{4} \mathrm{D}_{1} \mathrm{D}_{2} \mathrm{D}_{2} \mathrm{D}_{3} \mathrm{D}_{3} \mathrm{D}_{3} \mathrm{D}_{1} \mathrm{D}_{1} \mathrm{D}_{5} \mathrm{D}_{5} \mathrm{D}_{5}$

3 strings, 5 disks and a window size of 5

\begin{tabular}{|l|l|l|l|l|l|}
\hline \multicolumn{1}{|c|}{ Disks-> } & 1 & 2 & 3 & 4 & 5 \\
\hline String1 & 1 & 0 & 1 & 1 & 1 \\
\hline String2 & 0 & 1 & 0 & 0 & 1 \\
\hline String3 & 0 & 1 & 1 & 0 & 0 \\
\hline
\end{tabular}

n x d Matrix

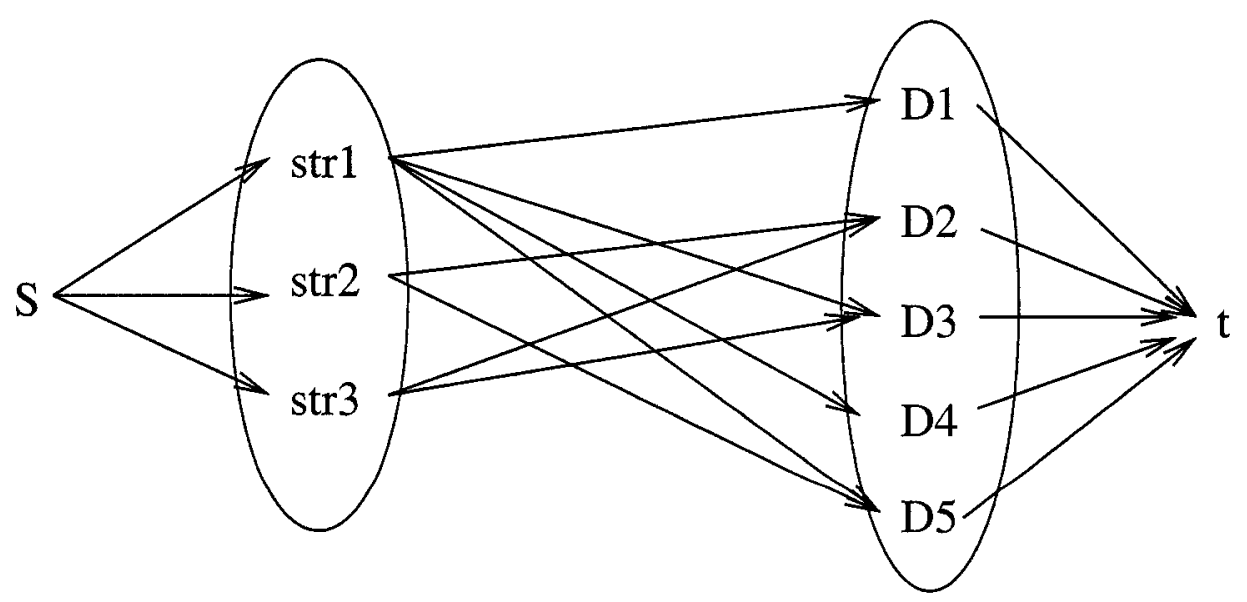

Augmented bi-partite graph representing the nxd marix

Figure 4.1 : Mapping from disk scheduling to flow 
to strings might get changed to make this possible, but the number of disks assigned to any other string will not change by this reassignment. Thus the weight of the fetch vector is increased without disturbing the relative allocations needed for fairness. The resource graph is modified to reflect the new assignments as described below. This step will be referred to as path conditioning in the description of the algorithms.

Path Conditioning: In the current resource graph every edge $\left(R_{i}, D_{j}\right)$ in the discovered path is replaced by its reverse edge $\left(D_{j}, R_{i}\right)$, and similarly every edge $\left(D_{s}, R_{t}\right)$ in the path is replaced by the reverse edge $\left(R_{t}, D_{s}\right)$. The last edge on the discovered path from some $D_{u}$ to $t$ is also replaced by the reverse edge $\left(t, D_{u}\right)$.

The invariant maintained by the algorithm is that the presence of an edge $\left(D_{s}, R_{t}\right)$ in the resource graph at the start of an iteration means that currently disk $D_{s}$ is assigned to string $R_{t}$. Suppose the path going through selected node $R_{u}$ at this iteration is $\left(s, R_{u}, D_{j_{1}}, R_{j_{1}}\right.$, $\left.D_{j_{2}}, R_{j_{2}}, \cdots, D_{j_{k}}, R_{j_{k}}, D_{j_{k+1}}, t\right)$. This implies that at the start of the iteration there were k assignments: disk $D_{j_{i}}$ was assigned to string $R_{j_{i}}, 1 \leq i \leq k$. When the edges between string and disk nodes are reversed, the $k+1$ new assignments will be: $D_{j_{1}}$ to $R_{u}, D_{j_{2}}$ to $R_{j_{1}}$, and so on ending with $D_{j_{k+1}}$ assigned to $R_{j_{k}}$. Note that for each of the string vertices $R_{j_{i}}$ only the identity of the disk was changed. and not the number of disks assigned to them.

\subsubsection{Why maxflow, bipartite matching algorithms don't work?}

Although related to the problems of bipartite graph matching and determining maximum flow [CLR89], there are subtle differences, that preclude direct application of either of these algorithms to our problem. Modeling our problem as a flow problem and searching the space using calls to a max flow algorithm will require an exponential number of such calls. Similarly bipartite matching will produce a one-to-one rather than a many-to-one map between reference strings and disks; attempting to use repeated applications of bipartite matching cannot guarantee that a fair assignment will be found. We present pathological cases to show that bipartite matching and maxflow algorithms don't solve the problem at hand. 


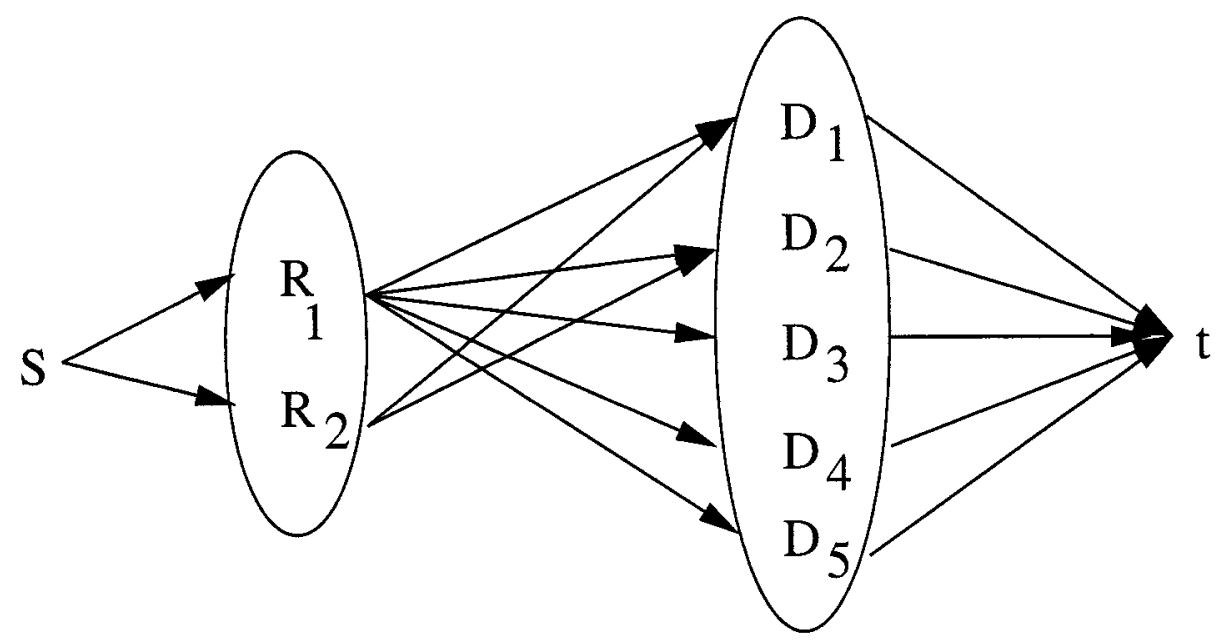

Figure 4.2: A pathological case for Iterative bipartite matching

Bipartite matching:Consider the example shown in Figure 4.2, with 2 reference strings and 5 disks. If we run the maximum bipartite matching algorithm on the simple problem described in Figure 4.2 the algorithm will give the flow as $\left\{R_{1} \mapsto D_{1}, R_{2} \mapsto D_{2}\right\}$ after first iteration. Then we remove the matched $D_{i}$ (disk nodes) from the graph and find a matching again. In the next iteration it will give the flow as $\left\{R_{1} \mapsto D_{3}, R_{2} \mapsto n o t h i n g\right\}$. But still number of blocks scheduled is 3 , that is less than 5 . Then again in the next iteration the flow will be $\left\{R_{1} \mapsto D_{4}, R_{2} \mapsto\right.$ nothing $\}$ and finally the flow will be given as $\left\{R_{1} \mapsto D_{5}\right\}$. The algorithm assigns 4 disks to string 1 and 1 disk to string 2 . The fetch vector $F=[4,1]$ which is lexicographically larger than fetch vector [3,2] which is possible in the given example by assigning $D_{3}, D_{4}, D_{5}$ to $R_{1}$ and $D_{1}, D_{2}$ to $R_{2}$. The problem with iterative bipartite matching is that the assignments once made in an iteration cannot be modified in future iterations.

Ford Fulkerson's maxflow algorithm: As we argued earlier that checking all possible flows require an exponential algorithm. We consider an example where sufficient flow value is assigned to each reference string node $\left(R_{i}\right.$ 's) and the maxflow is calculated. The number of disks assigned to $i^{\text {th }}$ string is equal to the flow passing through node $R_{i}$. Consider the example shown in Figure 4.3 with 3 reference strings and 7 disks. The flow assignment is as follows: 


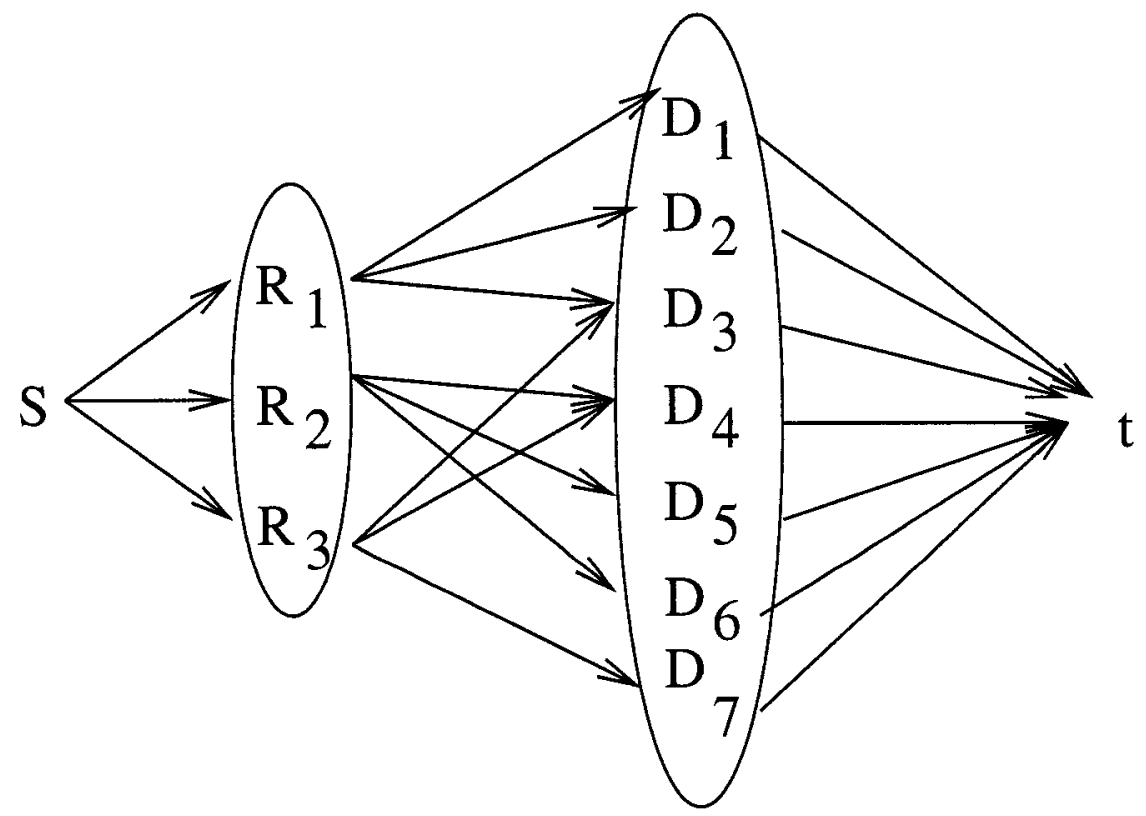

Figure 4.3 : A pathological case for maxflow algorithm

1. $\forall$ edges $\in E_{s}$, flow $=\mathrm{D}$

2. All the rest of the edges have unit flows.

The maxflow is limited by $\mathrm{D}$ which is the total incoming flow into $t$. The algorithm can find augmenting paths in a manner to assign flow values of 3 (allocating disks $D_{1}, D_{2}$ and $D_{3}$ ) to $R_{1}$, 3(allocating disks $D_{4}, D_{5}$ and $D_{6}$ ) to $R_{2}$ and 1 (disk $D_{7}$ ) to $R_{3}$, whereas the minimal lexicographic fetch vector can be $F=[3,2,2]$. Hence finding maxxflow doesn't ensure any sort of fairness unless there is some kind of fairness notion built into finding the augmenting paths and assignment of disk nodes to strings.

Our algorithm is inspired by the ideas of augmenting paths and residual graphs employed in the Ford Fulkerson algorithm for maximum flow in a network, but has been suitably refined for the problem at hand. 


\subsection{Local fairness}

Haritsa et al. [HP95] describe that fairness for a service provided by some resource to users can be achieved in 2 ways: instantaneous and statistical. For instantaneous fairness the resource is distributed equally among users at every instant of operation, whereas statistical fairness considers the distribution of resource among users during a certain window of time or for a certain number of requests. It isn't possible to define instantaneous fairness when there is a single resource that needs to be allocated to different users. Since we have multiple disks that need to be shared by multiple strings we can define instantaneous fairness as equal distribution of disks among strings in a single I/O step. Similarly statistical fairness requires equal distribution of total disk bandwidth among all reference strings during certain number of I/O's. We use the terms local and global in place of instantaneous and statistical respectively in this thesis but they mean the same. We define the local faimess problem formally as follows:

Problem definition: Find an assignment of disks to reference strings such that the fetch vector, $\mathrm{F}=\left[b_{1}, b_{2}, \ldots, b_{n}\right]$ has maximal weight and is lexicographically minimum.

In the resource graph $\mathrm{G}$ defined earlier let $\hat{D}$ be the number of disk nodes that have at least one incident edge. Then the maximal weight of the fetch vector is $\hat{D}$, i.e. a work-conserving schedule will assign $\hat{D}$ disks.

\subsubsection{LFS algorithm}

A straightforward algorithm to obtain local faimess can try all assignments made up of ordered partitions of $\hat{D}$ into $n$ components, in increasing lexicographic order until a feasible schedule is found. However, the running time of such an algorithm would be exponential as there are $\theta\left(\hat{D}^{n}\right)$ possible partitions of $\hat{D}$ into $n$ parts, where the order of parts is important.

The algorithm LFS shown in Figure 4.4 gives the maximal weight, lexicographically minimum fetch vector in $\mathrm{O}((n+D) n D)$ time, where $n$ is the number of reference strings and $D$ is the number of disks. The algorithm has the structure described in overview. The priority vector begins with all components 0 . At every iteration the (non saturated) 
component with smallest value will have the highest priority in the vector $\mathcal{P}$. Once a node $R_{i}$ is selected based on values of $\mathcal{P}$, a path is searched from $\mathrm{s}$ to $t$ using the edge $\left(\mathrm{s}, R_{i}\right)$. The node $R_{i}$ is saturated if no path is found, otherwise the graph is changed dynamically using path conditioning as described in the overview. Hence at every iteration either the weight of the priority vector increases by 1 , or one component (string node) is saturated. The use of the dynamic graph created by the path conditioning step ensures that the assignments of disks at some stage do not preclude reassignment at a later stage. This observation and the pruning of saturated nodes allows the algorithm to terminate in no more than $\hat{D}+n$ iterations; each iteration requires an $\mathrm{O}(n \hat{D})$ time path searching algorithm.

We present some lemmas related to LFS algorithm to prove its complexity and correctness.

Lemma 4.1. Once a disk node $D_{i}$ has been assigned to some string node it will never become free (although it may be reassigned to a different string node).

Proof. At every iteration, a path is found from $s$ to $t$. If the disk $D_{i}$ is not in the path, it remains assigned to the same string and nothing happens to it. Now lets assume $D_{i}$ is in path. The path can only be of the form $\left(s, R_{u}, D_{j_{1}}, R_{j_{1}}, D_{j_{2}}, R_{j_{2}}, \cdots, D_{j_{k}}, R_{j_{k}}, D_{j_{k+1}}, t\right)$, and $D_{i}$ can be one of the disks in the path. Let $D_{i}$ be $D_{j_{m}}$ and it is currently assigned to $R_{j_{m}}$, then after the path conditioning step, it will be assigned to the string node preceding it in the path i.e. $R_{j_{m-1}}$. Hence any disk once assigned cannot become free.

Lemma 4.2. The number of disks assigned to a string never decreases.

Proof. At every iteration, the path can only be of the form $\left(s, R_{u}, D_{j_{1}}, R_{j_{1}}, D_{j_{2}}, R_{j_{2}}, \cdots\right.$, $\left.D_{j_{k}}, R_{j_{k}}, D_{j_{k+1}}, t\right)$ because there is no way to come back to $\mathrm{s}$ and only one node can have an edge to $t$, rest of the edges hop between string nodes and disk nodes using the back edges added due to path conditioning step. Whenever a disk $D_{j_{m}}$ is reassigned from $R_{j_{m}}$ to $R_{j_{m-1}}$, the node $R_{j_{m}}$ gets disk $D_{j_{m+1}}$. Hence none of the strings loose any count of disks assigned to it. 
Lemma 4.3. If a string node $R_{i}$ is saturated then no path can be found through it even in future iterations.

Proof. $R_{i}$ is saturated when no path is found through it. Let $u_{d}$ and $u_{s}$ be the set of disk and string nodes reachable from $R_{i}$. Since there is no path, that means for all $D_{i} \in u_{d}$, no edge $\left(D_{i}, \mathrm{t}\right)$ exists and this will remain true in future by Lemma 4.1. Also none of the string nodes $R_{j} \in u_{s}$, have an edge to a disk node that is not assigned till now because a path would be found otherwise. Any of the incoming/outgoing edges for a node changes only if that node is in a path from $s$ to $t$ during path conditioning step. Since nodes in $u_{d}$ and $u_{s}$ cannot be on a path unless some edges change, they will never be on a path in future iterations, if there is no path in the current iteration.

Lemma 4.4. LFS finds the minimum lexicographical allocation of $\hat{D}$ disk blocks to reference strings.

Proof. We show the correctness of LFS by induction on number of saturated nodes. Let number of saturated nodes be $k$ at any iteration. The value of $k$ increases monotonically during the iterations of the LFS algorithm and there is no need to reconsider saturated nodes for path search (Lemma 4.3). Let fetch vector $F=\left[f_{a_{1}}, f_{a_{2}}, \cdots, f_{a_{n}}\right]$, where $f_{a_{i}}>f_{a_{i+1}}$ for all values of $i$.

1. Base case: Initially $k=0$, and the fetch vector $F=[0,0, \cdots, 0]$, which is minimum with weight zero.

2. Hypothesis: Let $F$ be the minimum fetch vector, with $k$ saturated components and some weight $w$. Now we will show that it will be the minimum lexicographic vector even when number of saturated nodes become $k+1$.

Note that the algorithm always picks the minimum non-saturated component $f_{i}$ and tries to find a path through corresponding string node $R_{j}$. As soon as a component is saturated, its position in the fetch vector never changes. Thus the component that saturates first will be last in the vector, the component that saturates second will be 
second from last and so on. Hence $k$ saturated components will be from $f_{a_{n-k+1}}$ to $f_{a_{n}}$.

In the current iteration we choose an unsaturated string node $R_{i}$ with the minimum component $f_{a_{n-k}}$. We search for a path through $R_{i}$ and there are two possibilities: (1) if no path is found, the node $R_{i}$ is saturated and the corresponding component $f_{a_{n-k}}$ is freezed and it stays the same in final fetch vector. This is because there will not be any path through $R_{i}$ even in future by Lemma 4.3 . The number of saturated nodes is increased to $k+1$. Hence the vector remains lexicographic minimum with $k+1$ saturated nodes. (2) if a path is found, then $f_{a_{n-k}}$ increases by one and we again have a minimum fetch vector with weight increased by one unit. This is because $f_{a_{n-k}}$ was minimum component and other components do not change by Lemma 4.2.

Lemma 4.5. The running time of LFS algorithm is $O((n+D) n D)$.

Proof. At every iteration, either a node $R_{i}$ gets saturated or the weight of the vector increases by 1 . Maximum value of weight can be $D$ and number of nodes that can be saturated is $n$. Hence there can be no more than $(n+D)$ iterations. The complexity of any iteration can be $O(n D)$, which is the complexity of finding a path from $s$ to $t$ using DFS (depth first search). Hence the total complexity can be $O((n+D) n D)$.

\subsection{Global Fairness and QoS Scheduling}

The local faimess metric aims to distribute disk accesses fairly at every I/O step. No history of block accesses is kept by the algorithm. There are situations where such an approach may be inadequate. For instance, if an application has a burst of requests to just a few disks, it will receive a smaller fraction of the bandwidth at each I/O step comparable to applications whose accesses are spread over a larger number of disks. In this case it may be desirable to favor these strings when the hot spot activity passes. In other cases a systematic 
(1) LFS(Locally Fair Scheduling) algorithm:

(2) weight $=0$

(3) $\mathrm{G}$ is the augmented bipartite resource graph, with $E_{s}=\phi$

(4) Let priority vector $\mathcal{P}=[0,0, \ldots, 0]$.

Mark all elements of the vector as non-saturated.

(5) while (weight $<\hat{D}$ )

(6) Choose the lowest-valued non-saturated element $p_{i}$ of $P$ with ties broken arbitrarily. Add an edge $\left(s, R_{i}\right)$

(7) Find any path from $s$ to $t$ that includes the edge $\left(s, R_{i}\right)$.

(8) if (no path is found)

(9) $\quad$ Mark $p_{i}$ as saturated in $P$.

(10) else

(11) $\quad$ weight $=$ weight $+1 ; p_{i}=p_{i}+1$

(12) Update graph $G$ by path conditioning

Figure 4.4: $\mathrm{O}((n+D) n D)$ algorithm for local fair scheduling 
but small difference in local allocation at each step, may result in significant spread of the cumulative bandwidth allocation for long running applications. In order to handle such situations we define a global fairness criterion, and describe how we can adapt our basic algorithm for this purpose. Intuitively the globally-sensitive algorithm called GFS, keeps the history of accesses in the cumulative fetch vector $\mathrm{CF}$. In the current $\mathrm{I} / \mathrm{O}$ assignment, GFS will try and equalize the components of CF, by favoring strings which are lagging in the total number of blocks fetched so far.

The formal definition of the algorithm is given in Figure 4.5. As in LFS, weight tracks the number of disks allocated at any step of the algorithm. We let $C F=\left[B_{1}, B_{2}, \cdots, B_{n}\right]$ be the $\mathrm{CF}$ vector at the start of the I/O step. Initialize the priority vector $\mathcal{P}=\left[p_{1}, p_{2}, \cdots, p_{n}\right]$, where $p_{i}=B_{i}$. Intuitively $p_{i}$ keeps track of the total number of blocks fetched by the $i^{t h}$ string. In the main loop, strings are scheduled in increasing order of blocks accessed, so that strings which have the largest slack(or minimum $B_{i}$ ) at any step are given a chance to catch up. The rest of the algorithm stays the same. The running time of GFS is also $\mathrm{O}((\mathrm{n}+\mathrm{D}) \mathrm{nD})$ based on the reasoning similar to the one given for LFS.

\subsubsection{Weighted allocation}

For QoS, we want to assign unequal proportions of resources to the tasks needing them. This distribution is quite common in today's economy where customers are served and given guarantees according to their financial capabilities. We also need to enable a differentiated distribution of resources to the tasks based on their priorities. Some real world examples are: different multimedia streams requiring different disk bandwidth based on the quality of picture, flows in the network requiring different bandwidth based on the QoS guarantees given to them, or tasks running on a processor are assigned a priority. We study the problem of assigning disk bandwidth in a skewed manner to multiple reference strings. Let the proportionate vector $V_{p}=\left[a_{1}, a_{2}, \cdots, a_{n}\right]$, where $a_{i}=B_{i} / w_{i}$ for $i^{t h}$ string . Intuitively $V_{p}$ shows the proportionate bandwidth allocated to each string and we want fairness among the components of $V_{p}$. The algorithm for weighted allocation called $\mathrm{W} e \mathrm{AB}$, keeps 


\section{(1) GFS (Global Fair Scheduling) algorithm:}

(2) weight $=0$

(3) $\mathrm{G}$ is the augmented bipartite resource graph, with $E_{s}=\phi$

(4) Let priority vector $\boldsymbol{P}=\left[p_{1}, p_{2}, \ldots, p_{n}\right]$, where $p_{i}=B_{i}$. Mark all $p_{i}$ 's as non-saturated

(5) while (weight $<\hat{D}$ )

(6) Choose the smallest non-saturated element $p_{i}$ of $\mathcal{P}$ with ties broken arbitrarily. Add the edge $\left(s, R_{i}\right)$

(7) Find any path from $\mathbf{s}$ to $\mathbf{t}$ that includes the edge $\left(s, R_{i}\right)$.

(8) if(no path is found)

(9) Mark $p_{i}$ as saturated in $\mathcal{P}$.

(10) else

(11) weight $=$ weight $+1 ; B_{i}=B_{i}+1 ; p_{i}=p_{i}+1$

(12) Update graph $G$ by path conditioning

Figure 4.5 : $\mathrm{O}((n+D) n D)$ algorithm for global fair scheduling 
the history of accesses in the cumulative fetch vector CF. Initialize the priority vector $P=$ $\left[p_{1}, p_{2}, \cdots, p_{n}\right]$ as $p_{i}=B_{i} / w_{i}$. The Priority vector is initialized to indicate the proportionate bandwidth given to the $i^{t h}$ string so far. In the current $\mathrm{I} / \mathrm{O}$ step, WeAB will assign disks to try and equalize the components of $P$, by favoring strings which are lagging in their proportion of blocks fetched so far. Note that assigning a disk to $R_{i}$ increases $p_{i}$ by $1 / w_{i}$ instead of 1. In the main loop, strings are scheduled in decreasing order of proportionate slack, so that strings which have the largest proportionate slack at any step are given a chance to decrease their slack. Mathematically, the string with the minimum value of $\left(B_{i}+1\right) / w_{i}$ has the highest priority and is chosen to explore the existence of a path. At each step either a string node gets saturated or an additional disk is scheduled. It continues until $\hat{D}$ disks are scheduled. A more formal description of the algorithm is given in Figure 4.6.The WeAB algorithm also runs in $\mathrm{O}((\mathrm{n}+\mathrm{D}) \mathrm{nD})$ time.

\subsection{Simulation Results}

In this section we present the results of evaluating LFS, GFS and WeAB using simulation on synthetically generated workloads. We consider an idealized situation where we assume that each request consumes equal amount of resources. Either the service time of requests is known a priori or we know some average value that can be used whenever we are doing the allocation. Hence the resources are allocated in discrete units and consumed in a synchronous fashion. Therefore simple measures such as number of $\mathrm{I} / \mathrm{Os}$ or number of blocks accessed are used to evaluate this situation. The results show that our algorithms obtain fairness and weighted QoS very accurately.

We use two modes of generating workloads in this evaluation. A reference string is composed of requests generated by either one or both modes of generation. The two modes are as follows:

1. Random: In this model every request in a string belongs to a disk chosen randomly with equal probability. 
(1) WeAB(Weighted Allocation of Bandwidth) algorithm:

(2) weight $=0$

(3) $\mathrm{G}$ is the augmented bipartite resource graph with $E_{s}=\phi$

(4) Let priority vector $P=\left[p_{1}, p_{2}, \cdots, p_{n}\right]$, where $p_{i}=B_{i} / w_{i}$. Mark all $p_{i}$ 's as non-saturated

(5) while (weight $<\hat{D})$

(6) Choose the non-saturated element $p_{i}$ of $\mathcal{P}$ such that $\left(B_{i}+1\right) / w_{i}$ is minimum with ties broken arbitrarily. Add an edge $\left(s, R_{i}\right)$

(7) Find any path from $\mathbf{s}$ to $t$ that includes the edge $\left(s, R_{i}\right)$.

(8) if(no path is found)

(9) $\quad$ Mark $p_{i}$ as saturated in $\mathcal{P}$.

(10) else

(11) weight $=$ weight $+1 ; B_{i}=B_{i}+1 ; p_{i}=B_{i} / w_{i}$

(12) Update graph $G$ by path conditioning

Figure 4.6: $\mathrm{O}((\mathrm{n}+\mathrm{D}) \mathrm{nD})$ algorithm for weighted allocation 
2. Skewed: In this model the $i^{\text {th }}$ request belong to the same disk as the $(i-1)^{\text {th }}$ request with some probability $p ; p$ starts with a certain maximum value (close to 1 ) and keeps on decreasing linearly. This produces skewed access patterns for reference strings, where there are bursts of requests to the same disk, followed by a burst on another disk, and so on. This essentially captures the locality in requests of various applications.

In the simulation, we show three basic characteristics of proposed algorithms. First is the ability to isolate the performance of various clients from each other, the second to show that the algorithms do achieve weighted allocation of resources, and the third is to verify their work conserving nature.

\subsubsection{Random model}

To test the behavior of LFS and GFS algorithms, we performed an experiment with 32 disks, and 5 reference strings of equal weights all generated using random model. Figure 4.7 and Figure 4.8 show the distribution of bandwidth obtained by each of the strings as a function of the I/Os number, for the LFS and GFS algorithms respectively. The bandwidth for a reference string $i$, at any $\mathrm{I} / \mathrm{O}$ is simply the fraction of fetched blocks that belong to string $i$. We observe that both algorithms perform quite well in allocating the fair amount (20\%) of the bandwidth to each string. LFS algorithms causes small fluctuations at each IO step, because it measures the instantaneous allocation that is sensitive to the random distribution of requests (Figure 4.7); in case of GFS, the history of blocks served per reference string averages out the fluctuations over a large time window. The small fluctuations in the beginning of Figure 4.8 are related to the distribution of disk requests in strings and occur due to the work conserving nature of the algorithms.

For weighted allocation of bandwidth, we performed an experiment with 4 randomlygenerated reference strings and 64 disks. Reference strings $R_{1}, R_{2}, R_{3}$ and $R_{4}$ have been assigned relative priorities of $0.4,0.3,0.2$ and 0.1 respectively. Figure 4.9 shows the actual bandwidth allocated to the strings using the $\mathrm{W} e \mathrm{AB}$ algorithm; the bandwidth allocated to 


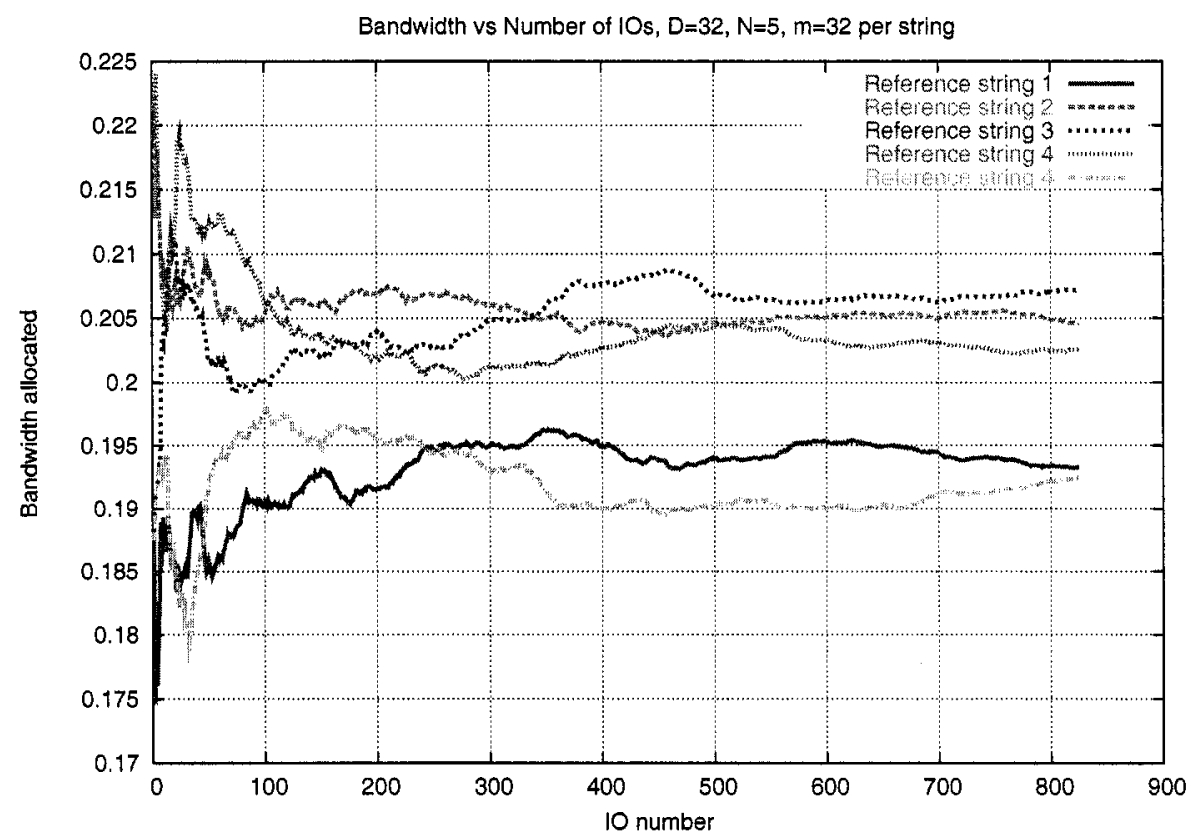

Figure 4.7 : Output of LFS for 5 strings

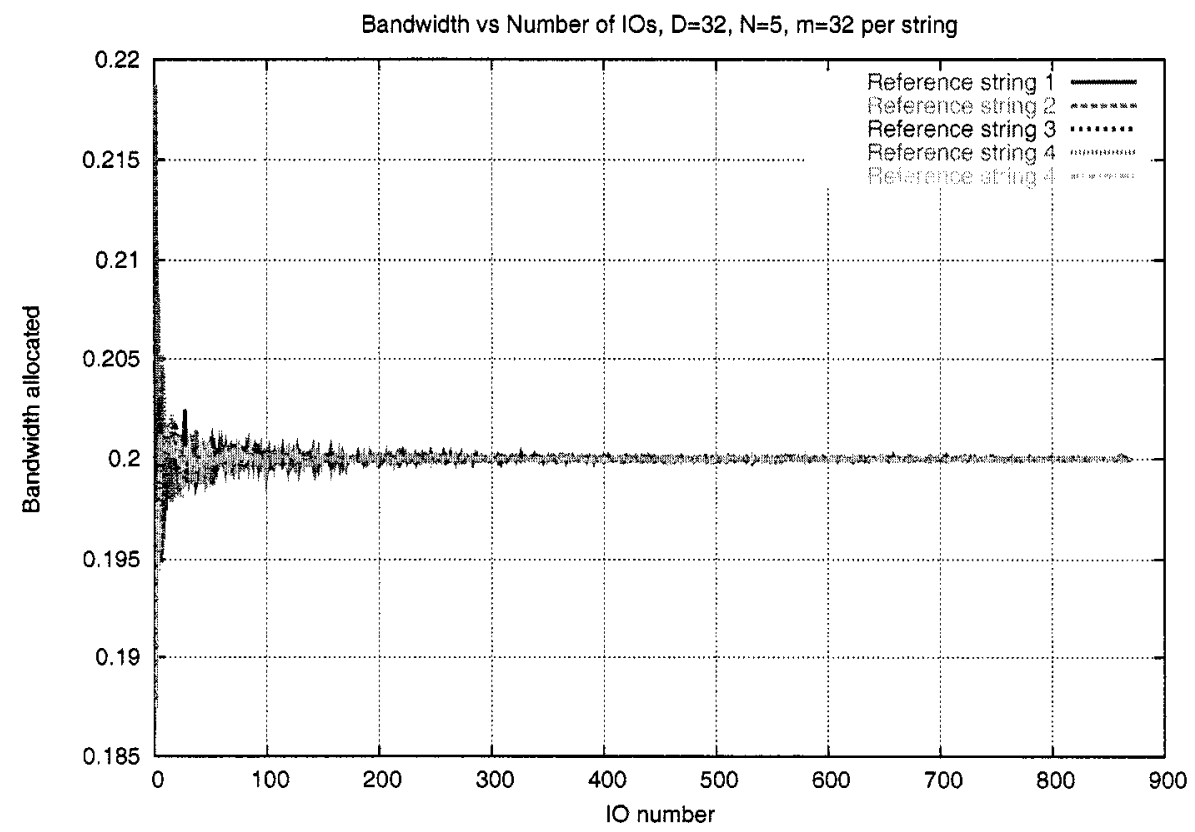

Figure 4.8 : Output of GFS for 5 strings 


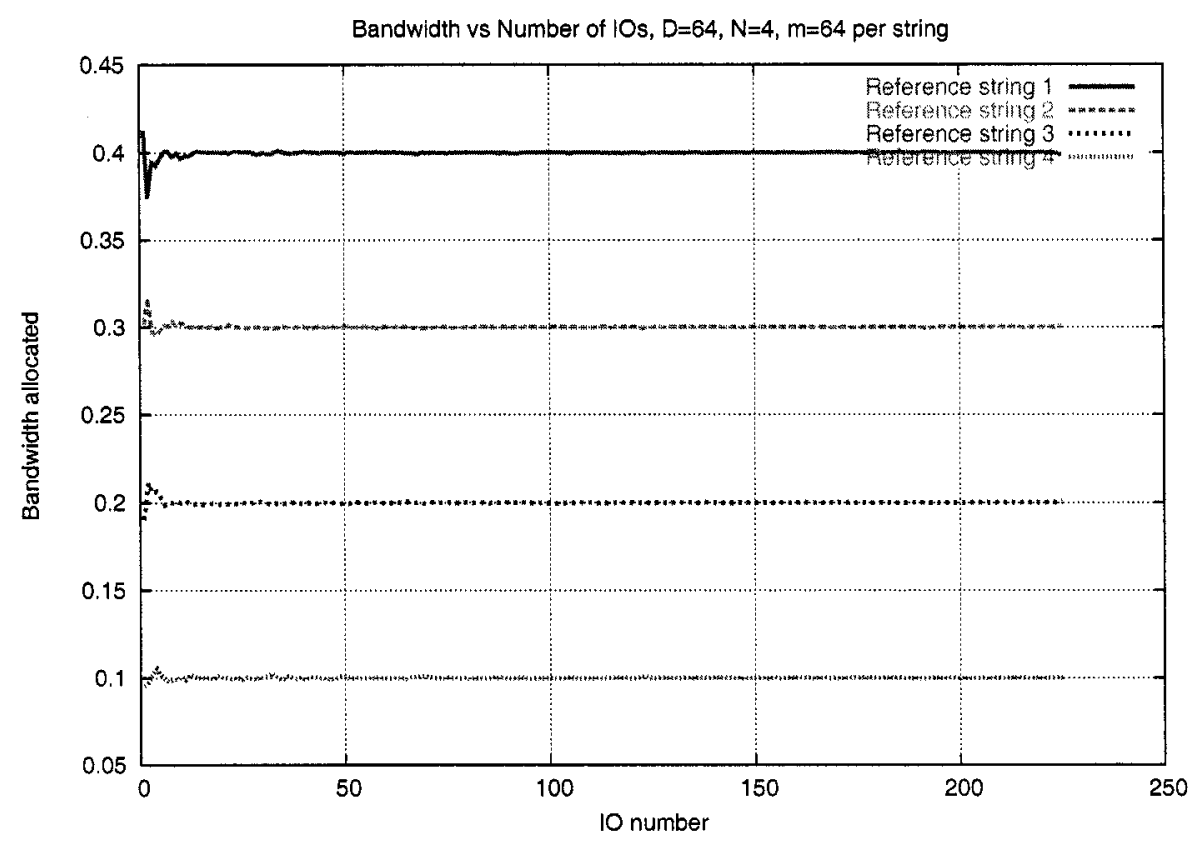

Figure 4.9 : WeAB with weights $0.4,0.3,0.2,0.1$

various strings is very close to the weights assigned to them, not surprisingly given the uniformly random distribution of accesses and the time averaging behavior of the $\mathrm{W} e \mathrm{AB}$.

\subsubsection{Skewed Input model}

In this section, we present results for testing our algorithms with the skewed input model. The skewed model tests the efficacy of the algorithms more rigorously. Workloads consists of inter-leaved regions where each region is generated in either a random or a skewed manner. For these experiments we chose, $\mathrm{D}=64$, and we experimented with 5 reference strings, where 3 were generated in a random manner. The other two were generated as follows: assuming the total length is $\mathrm{L}$, first $\mathrm{L} / 5$ requests are generated in the skewed manner, $\mathrm{L} / 5$ to $2 \mathrm{~L} / 3$ are generated in the random manner, $2 \mathrm{~L} / 3$ to $4 \mathrm{~L} / 5$ is generated again in the skewed manner and the rest is generated randomly. We chose these partitions to be able to show the difference in the behavior of LFS and GFS algorithms. We ran LFS algorithm on these 5 strings and the result is shown in Figure 4.10. Note that we plot blocks 


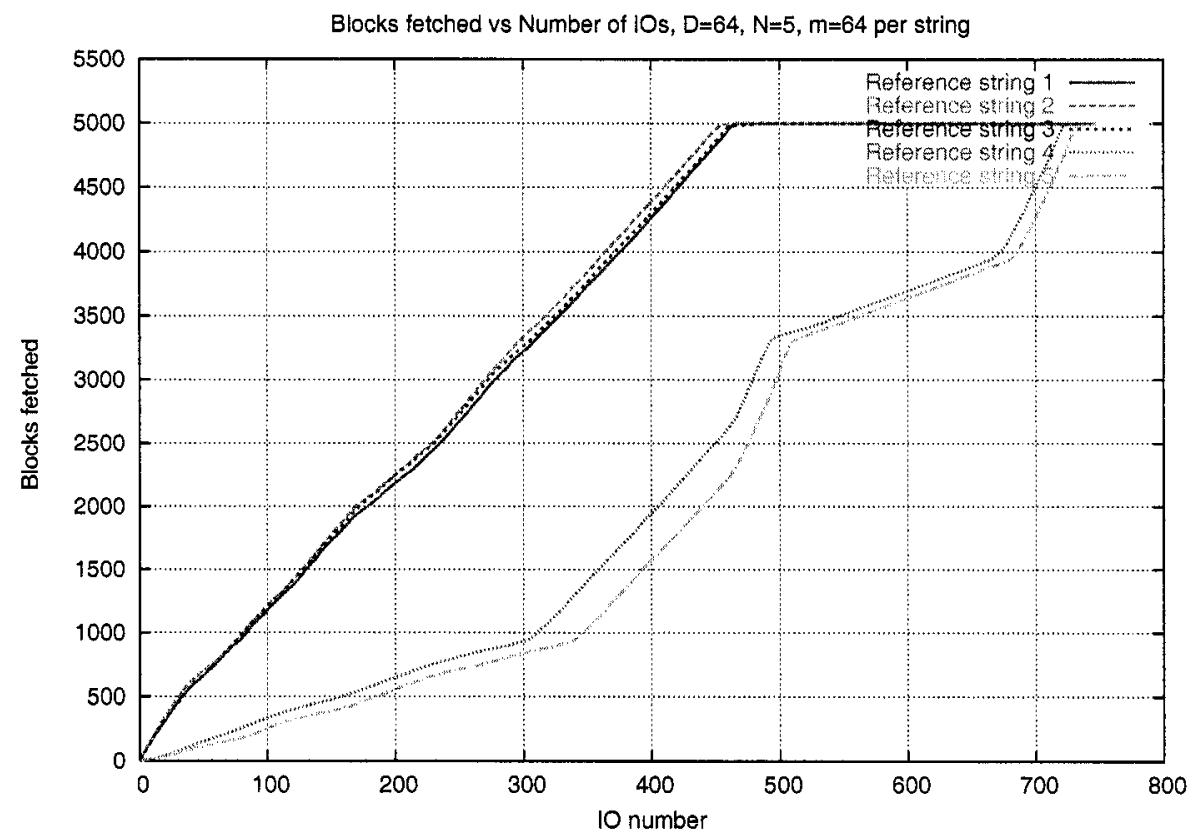

Figure 4.10 : LFS algorithm for skewed inputs

scheduled for each string on the $y$-axis and I/O number on the $\mathrm{x}$-axis. Thus the slope of the curve gives us blocks scheduled per I/O. We observe that till about $320 \mathrm{~J} / \mathrm{Os}$, strings 4 and 5 get fewer blocks per I/O because of their skewed nature. After that till IO number 480 , all reference strings get similar number of blocks per IO as expected by LFS. After 480, the slope for strings 4 and 5 increases slightly because strings 1, 2 and 3 are finished. From 500 to $680 \mathrm{IO}$, the second skewed region for strings 4 and 5 is reached, as shown by decreased slope. The rest of the region is generated with the random model and is scheduled with higher slope.

Figure 4.11 shows the results obtained by running GFS on a similarly constructed input. There are 4 major points to note here: (1) the skewed portion in strings 4 and 5 completes in about 280 IOs rather than 320 in case of LFS (2) the slope of curves 4 and 5 is much higher than the slope of curves 1, 2 and 3 because GFS favors the strings lagging behind in their fair share of blocks, and (3) around IO number 360 all strings have an equal number of cumulative blocks and their slope is equal from that point till strings 4 and 5 start having 


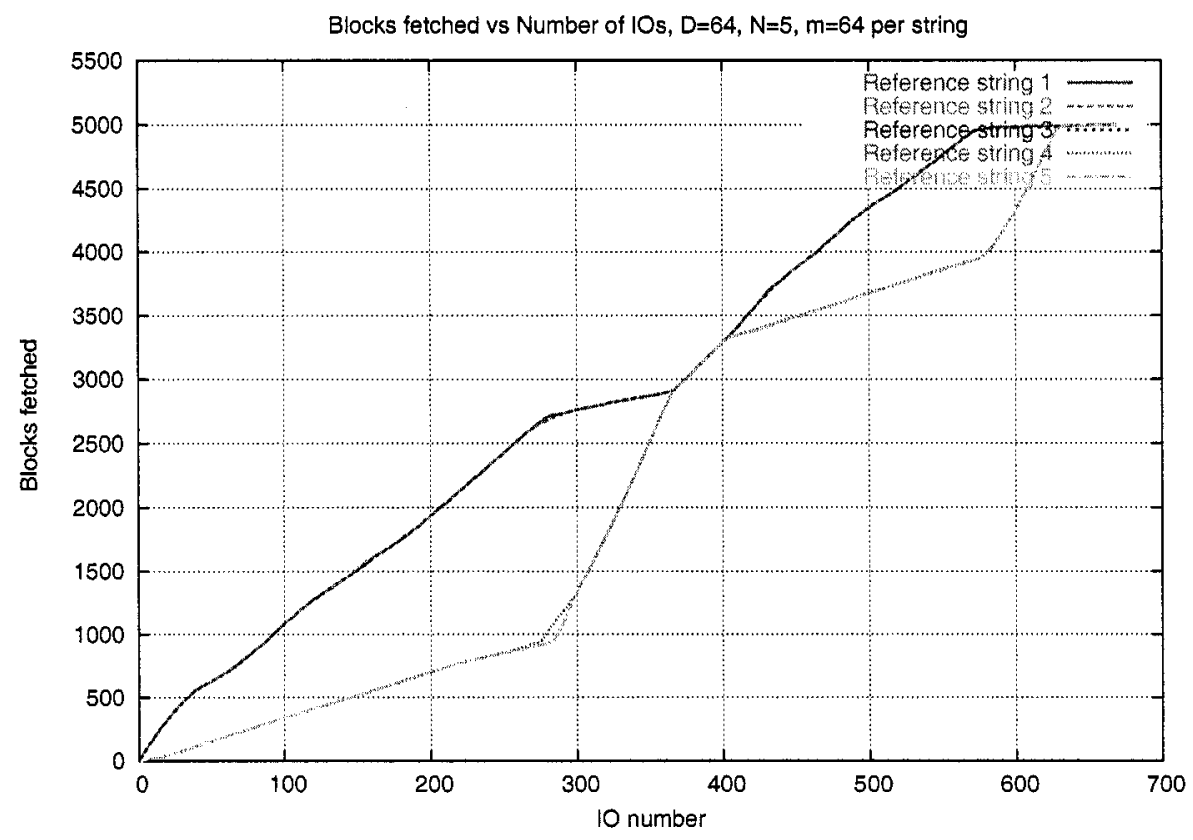

Figure 4.11 : Fair allocation by GFS (skewed)

skewed requests. (4) similar behavior happens when the skewed region comes again for 4 and 5 reappears.

To test $\mathrm{W} e \mathrm{AB}$ algorithm for weighted-allocation with skewed inputs, we experimented with 4 strings where 1 and 2 are generated randomly and 3 and 4 have an initial 5\% region generated in a skewed manner, and later a certain number of requests are skewed. The weights are assigned $0.1,0.2,0.3,0.4$ respectively to the strings. We observe that initially 3 and 4 get less than their weighted share due to the skewness, and 1 and 2 get more than their fair share due to the work conserving property. Also strings 2 gets larger share of the free capacity than 1 due to its higher weight, and 4 gets a larger share than 3 . As soon as the hot spot activity passes, strings approach their fair share of bandwidth. Later on near IO 2000 , there is again a short hot spot activity in string 4 that is shown by the slight fluctuation in the curves. Note that the free capacity is also distributed in a weighted manner. 


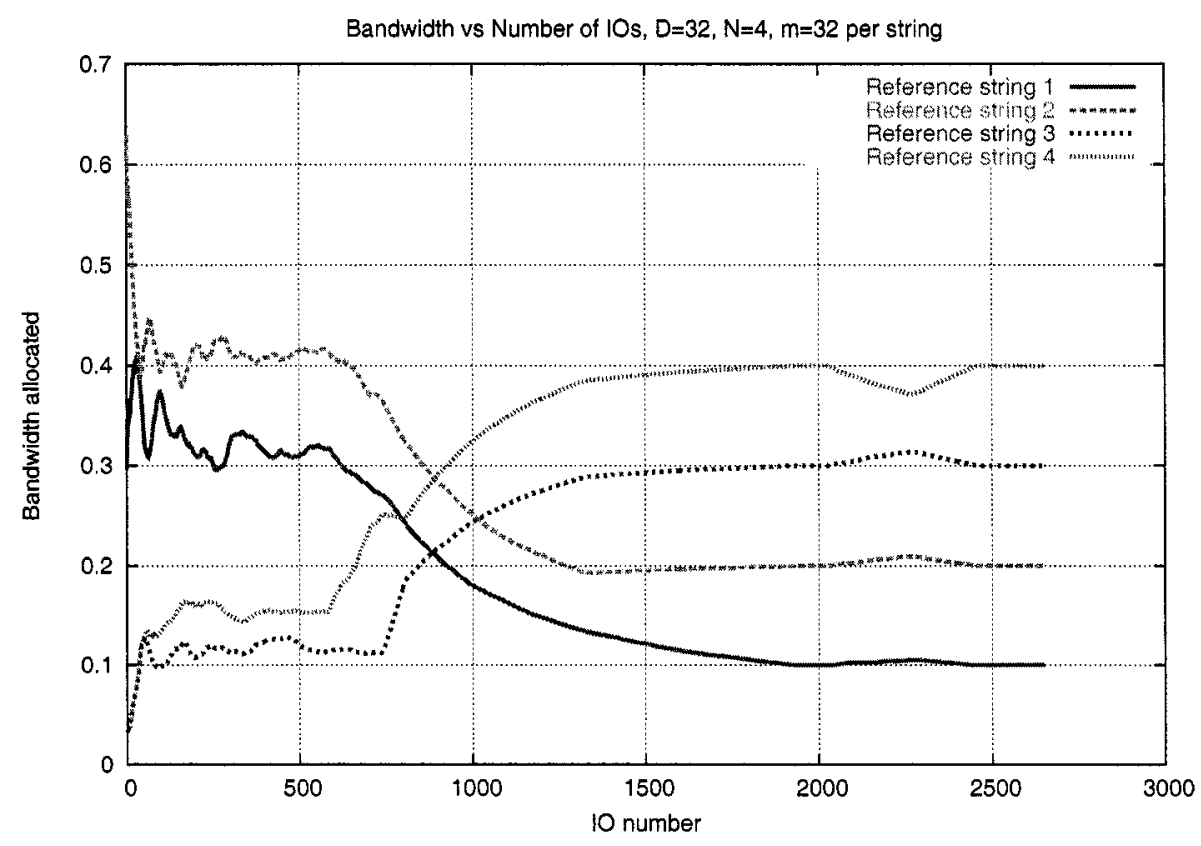

Figure 4.12 : Weighted allocation by $\mathrm{W} e \mathrm{AB}$ (skewed)

\subsubsection{Work conservation}

For work conservation, we experimented with 4 randomly generated reference strings, 64 disks and compared the throughput of GFS with the maximum number of blocks(D) that can possibly be scheduled in each LO. Figure 4.13 shows that GFS schedules number of blocks which is very close to maximum possible per IO. This is because all our algorithms are work conserving and they try to achieve maximal parallelism. Figure 4.14 shows the similar results when total number of blocks scheduled in N IOs is compared with maximum number $(D \times N)$ of blocks that can be scheduled.

\subsection{Related work}

Several disk scheduling policies have been proposed for single as well as multiple disks. Haritsa et al. [HP95] have presented efficient and fair scheduling algorithms for a single disk. They try to achieve fairness at the cylinder level by proposing strategies for disk head 


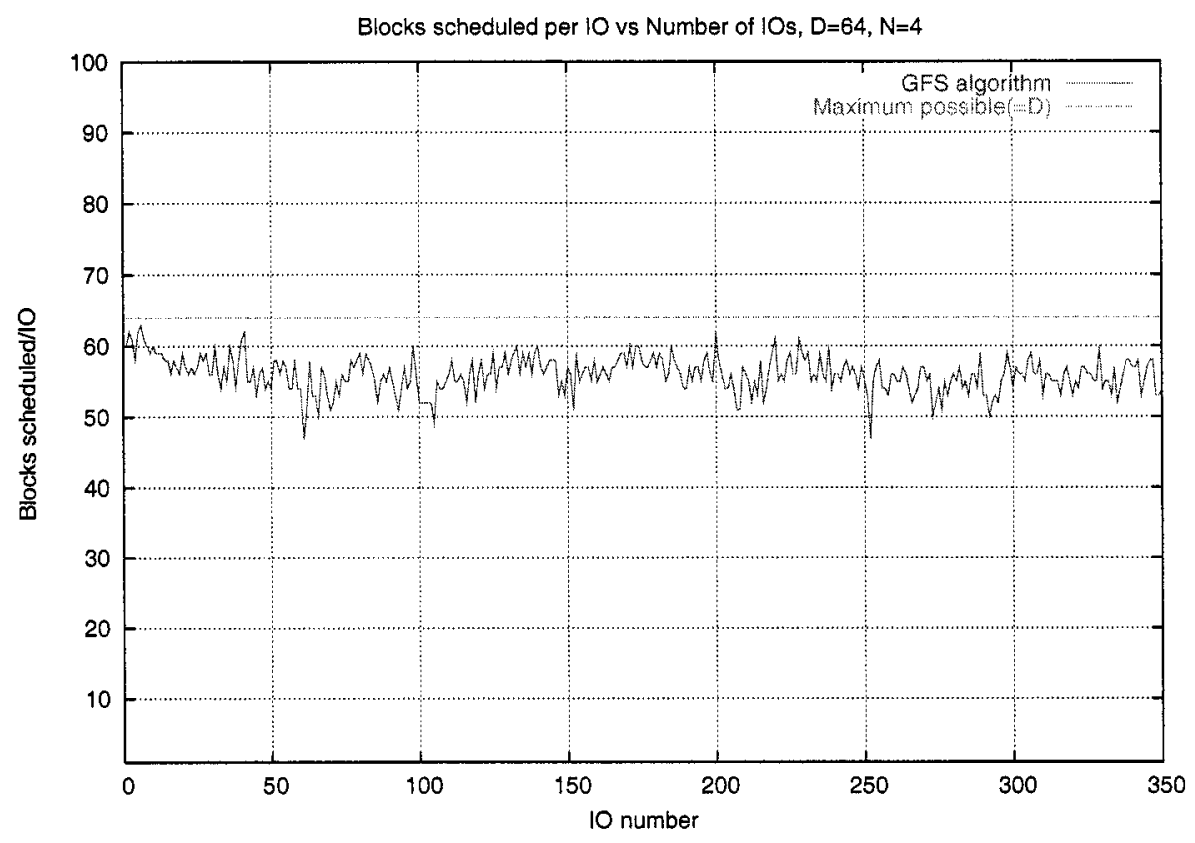

Figure 4.13 : GFS:blocks scheduled per IO vs D

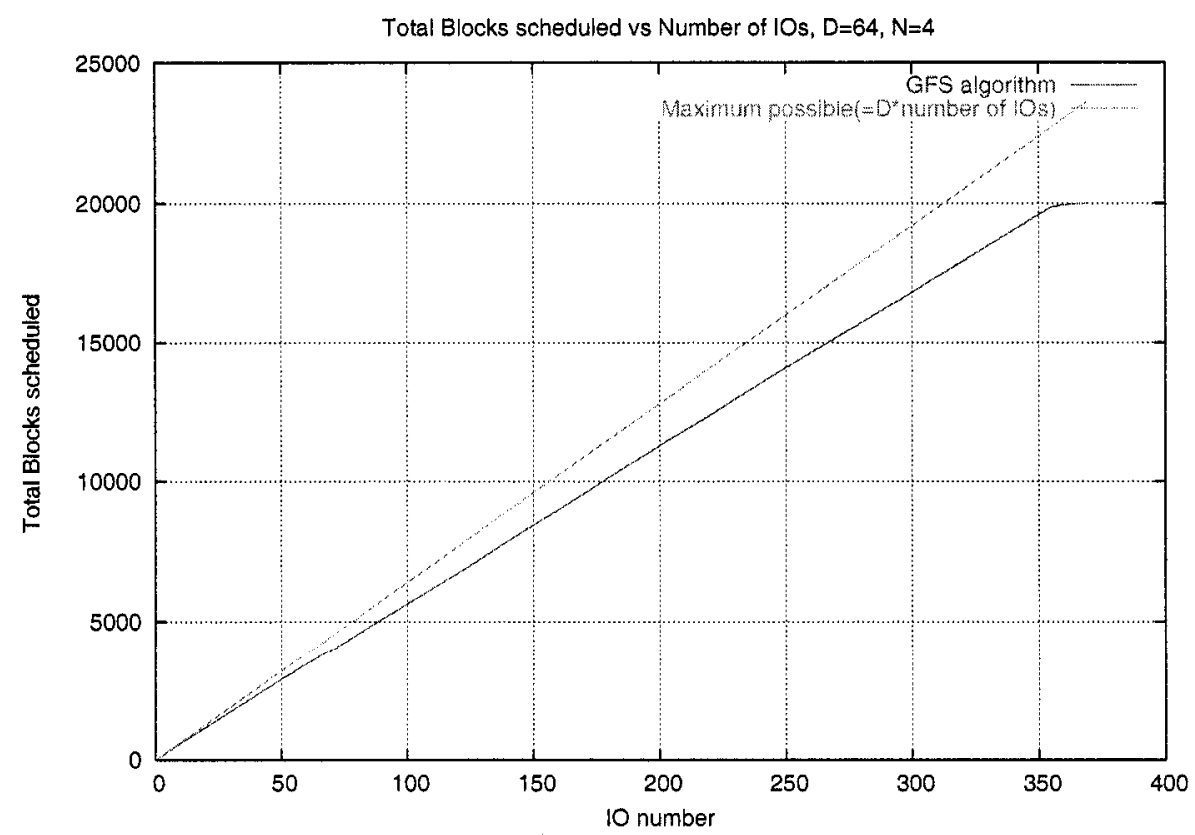

Figure 4.14 : GFS:total blocks scheduled in $n$ IOs vs $\mathrm{D}^{*} \mathrm{~N}$ 
movement after a request has been serviced. The YFQ [BBG $\left.{ }^{+} 99\right]$ algorithm which is an extension to generalized processor sharing(GPS) model of proportional resource allocation, allows applications to reserve a fixed share of disk bandwidth. Cello [SV98] provides a scheduling framework for a heterogeneous mix of applications accessing the disk. It employs a 2-level scheduling framework, where requests from applications are put into different queues based on their needs at first level and a common scheduler reorders the requests selected from these queues to minimize seek and rotational delays at second level. Our approach however, provides bandwidth allocation across multiple disks. By batching requests that belong to a single disk many of the lower level optimizations suggested in these works can be employed along with our higher level framework. For a single reference string over multiple disks Varman et al. [KV99a] presented an algorithm to get minimum length schedule. However the approach doesn't extend to multiple reference strings and the problem of finding the minimum length schedule for multiple reference strings over multiple disks was shown to be NP-complete in chapter 3. Jain et al. have proposed heuristics for parallel I/O [JSWB97]. They mapped the problem of allocating multiple I/O devices to various processors, to an edge coloring problem in a bipartite graph. Their solution aims to provide minimum length schedule with low execution overhead, without any notion of fairness or QoS.

Lumb et al. presented Facade [CLA03] that is an approach to fulfill Service Level Objective (SLO) of independent workloads accessing a storage system. Facade provides a dynamic trade-off between the IO rate and average latency by real-time scheduling and feedback based control of disk queue lengths. Their approach assumes the availability of a capacity planner for admission control. Our approach provides a finer control over allocation of disks to workloads in each I/O to achieve the desired bandwidth allocation.

Many schemes have been developed for fair scheduling in Internet routers. Schemes such as PIM [SOST93], iSLIP [McK99] and shakeup [GKR00] try to achieve high throughput and fairness by looking for maximal bipartite matching between inputs and outputs at each point of scheduling. iFS [NB02] provides faimess and QoS guarantees by assign- 
ing virtual start and finish times to each packet. These algorithms try to obtain a 1-to-1 mapping between input and output ports instead of the 1-to-many mapping required for our problem. Iteratively applying bipartite matching does not guarantee fair schedule in our case because it gives irrevocable mapping that cannot be changed in future iterations. Many other architectures have been proposed for differentiated allocation of bandwidth to flows such as diffservs [NBBB98] and QoSBox [CL01]. None of them can be used for QoS in automated storage systems [Wil01]. As stated in [Wil01], performance of storage systems depends a lot on the current state and storage protocols are not amenable to packet dropping and no support for traffic shaping exists in storage systems. Also applying Ford Fulkerson's maximum flow algorithm cannot achieve fairness unless flows are chosen very carefully. Example cases for the failure of these approaches are shown in chapter 4.

Automated tools such as Hippodrome [Aea02] and Minerva [Aea01] can be used to design storage systems, if the capacity requirements and workloads characteristics are known a priori. These tools go through the iterative process of designing and evaluating the system. Once the workloads or any other requirement changes the whole process needs to be repeated again. Our approach can handle short term fluctuations and provide weighted-QoS even in presence of unpredictable workloads. Fair bandwidth allocation for wireless [TS02] and wireline [Hah91] networks have received significant attention. The constraints are quite different in these cases. In case of wireline networks only users sharing the same link contend for the bandwidth and in case of wireless allocation users in a vicinity contend for bandwidth usage even if they use different wireless links. Most of the solutions have some kind of round robin scheme at each node that evolves dynamically with the reservations made by a flow and the current requirements of the flow. Such schemes are not applicable to disk scheduling where scheduling is centralized and not distributed. 


\section{Chapter 5}

\section{Conclusions and Future Work}

Conclusions. This thesis has described a number of scheduling mechanisms in parallel I/O systems. We explored the problem of achieving maximum throughput when multiple tasks are accessing the disk system and show that the problem is NP-complete. Knowing the hardness of the problem we suggested some heuristics for efficient scheduling and showed the worst case bounds for them. None of the heuristics performs better in the worst-case than the trivial algorithm of scheduling all the reference strings one after the another. We presented an optimal algorithm for efficient scheduling under restricted conditions.

In the later half of this thesis we turned our attention towards scheduling that can provide some QoS guarantees or fairness. We explored the scheduling schemes that can provide QoS guarantees in parallel I/O. LFS algorithm obtains local fairness at each I/O step. GFS algorithm provides fair allocation in a global way, by keeping a history of blocks accessed by each string and $\mathrm{W} e \mathrm{AB}$ is a very general scheme to obtain an $\mathrm{I} / \mathrm{O}$ schedule with weighted allocation of disk bandwidth to various strings. WeAB can support an economic model of services provided by a data center that need to provide differentiated bandwidth to various customers. We demonstrated via simulations that the $\mathrm{We} A \mathrm{~B}$ algorithm matches the bandwidth allocated to various application to their desired weights and GFS obtains a fair schedule. All these algorithms are work conserving (i.e. they never leave a disk unscheduled if it can be scheduled in a particular I/O) and provide high throughput.

\subsection{Future Work}

We plan to implement our scheduling algorithms in an actual system to get a better idea about performance and scalability of our approach in future. We are also looking for other 
resource allocation problems where these scheduling algorithms can be used. For optimal scheduling, we are looking for other restricted cases which can be shown NP-complete. More specifically is the problem with a fixed number of reference strings, with variable disks and memory buffers, Np-complete? This will help us in drawing a line that can divide tractable problems from intractable ones in parallel L/O. 


\section{Bibliography}

[Aea01] Guillermo A. Alvarez and et al. Minerva: an automated resource provisioning tool for large-scale storage systems. In ACM Transactions on Computer Systems, pages 483-518, November 2001.

[Aea02] Eric Anderson and et al. Hippodrome: running circles around storage administration. In File and Storage Technology (FAST'02), pages 175-188, January 2002 .

[AGL00] Susanne Albers, Naveen Garg, and Stefano Leonardi. Minimizing stall time in single and parallel disk systems. J. ACM, 47(6):969-986, 2000.

$\left[\mathrm{BBG}^{+}\right.$99] John Bruno, Jose Brustoloni, Eran Gabber, Banu Ozden, and Abraham Silberschatz. Disk scheduling with quality of service guarantees. In Proceedings of the IEEE International Conference on Multimedia Computing and Systems Volume II-Volume 2, page 400. IEEE Computer Society, 1999.

[BGV96] R. D. Barve, E. F. Grove, and J. S. Vitter. Simple randomized mergesort on parallel disks. Parallel Computing, 23(4):601-631, June 1996.

[BKVV00] R. D. Barve, M. Kallahalla, P. J. Varman, and J. S. Vitter. Competitive parallel disk prefetching and buffer management. Journal of Algorithms, 36:152-181, July 2000.

[BZ96] Jon C. R. Bennett and Hui Zhang. WF ${ }^{2} \mathrm{Q}$ : Worst-case fair weighted fair queueing. In INFOCOM (1), pages 120-128, 1996.

[BZ97] Jon C. R. Bennett and Hui Zhang. Hierarchical packet fair queueing algorithms. IEEE/ACM Transactions on Networking, 5(5):675-689, 1997. 
[CFKL95] P. Cao, E. W. Felten, A. R. Karlin, and K. Li. A study of integrated prefetching and caching strategies. In Proc. of the Joint Intl. Conf. on Measurement and Modeling of Computer Systems, pages 188-197. ACM Press, May 1995.

[CL01] Nicolas Christin and Jorg Liebeherr. The QoSbox: A PC-Router for quantitative service differentiation in ip networks. Technical report, University of Virginia, 2001.

[CLA03] Arif Merchant Christopher Lumb and Guillermo Alvarez. Façade: Virtual storage devices with performance guarantees. File and Storage technologies (FAST'03), pages 131-144, March 2003.

[CLR89] T. H. Cormen, C. E. Leiserson, and R. L. Rivest. Introduction to Algorithms. McGraw-Hill Book Company and The MIT Press, 1989.

[Coo71] Stephen Cook. The complexity of theorem-proving procedures. 3rd annual ACM symp. on Theory of Computing, pages 151-158, 1971.

[DAPW94] M. D. Dahlin, T. E. Anderson, D. A. Patterson, and R. E. Wang. Cooperative caching: Using remote client memory to improve file system performance. In Proc. of the First Symp. on Operating Systems Design and Implementation, pages $267-280,1994$.

[GJ79] Michael R. Garey and David S. Johnson. Computers and Intractability, A Guide to the Theory of NP-Completeness. W. H. Freeman and Company, 1979.

[GJS76] Michael R. Garey, David S. Johnson, and Ravi Sethi. The complexity of flowshop and jobshop scheduling. Mathematics of Operations research, 1:117129, May 1976.

[GKR00] M. W. Goudreau, S. G. Kolliopoulos, and S. B. Rao. Scheduling algorithms for input-queued switches:randomized techniques and experimental evaluation. IEEE INFOCOM, 3:1634-1643, March 2000. 
[Gol94] S. Golestani. A self-clocked fair queueing scheme for broadband applications. In INFOCOMM'94, pages 636-646, April 1994.

[GS78] Teofilo Gonzalez and Sartaj Sahni. Flowship and jobshop schedules: Complexity and approximation. Operations research, 26:36-52, January 1978.

[GVC96] Pawan Goyal, Harrick M. Vin, and Haichen Cheng. Start-time fair queuing: A scheduling algorithm for integrated servicespacket switching networks. Technical Report CS-TR-96-02, UT Austin, January 1996.

[Hah91] Ellen L. Hahne. Round-robin scheduling for max-min fairness in data networks. IEEE Journal of Selected Areas in Communications, 7, September 1991.

[HP95] J. R. Haritsa and T. Pradhan. Fair disk schedulers for high performance computing systems. Proc. of Inetrnational Conf. on High Performance Computing, December 1995.

[HPC04] Lan Huang, Gang Peng, and Tzi-Cker Chiueh. Multi-dimensional storage virtualization. SIGMETRICS Perform. Eval. Rev., 32(1):14-24, 2004.

[HSV01] D. A. Hutchinson, P. Sanders, and J. S. Vitter. Duality between prefetching and queued writing with parallel disks. In Ninth European Symp. on Algorithms, number 2161 in LNCS, pages 62-73. Springer, 2001.

[Jaf81] J. M. Jaffe. Bottleneck flow control. IEEE Trans. Comm., 29(7):954-962, July 1981.

[JCK04] Wei Jin, Jeffrey S. Chase, and Jasleen Kaur. Interposed proportional sharing for a storage service utility. SIGMETRICS Perform. Eval. Rev., 32(1):37-48, 2004. 
[JSWB97] Ravi Jain, Kiran Somalwar, John Werth, and J. C. Browne. Heuristics for scheduling I/O operations. IEEE Transactions On Parallel and Distributed Systems, pages 310-320, March 1997.

[Ka197] M. Kallahalla. Competitive prefetching and buffer management for parallel I/O systems. Master's thesis, Rice University, Dept. of Electrical and Computer Engr., May 1997.

[Ka100] M. Kallahalla. Phd thesis. Master's thesis, Rice University, Dept. of Electrical and Computer Engr., 2000.

[KCF $\left.{ }^{+} 96\right]$ Tracy Kimbrel, Pei Cao, Edward W. Felten, Anna R. Karlin, and Kai Li. Integrated parallel prefetching and caching. In Proceedings of the 1996 ACM SIGMETRICS International Conference on Measurement and Modeling of Computer Systems, pages 262-263. ACM Press, 1996.

[KJ99] David Kotz and Ravi Jain. I/O in parallel and distributed systems. In Allen Kent and James G. Williams, editors, Encyclopedia of Computer Science and Technology, volume 40, pages 141-154. Marcel Dekker, Inc., 1999.

[KK00] Tracy Kimbrel and Anna R. Karlin. Near-optimal parallel prefetching and caching. SIAM J. Comput., 29(4):1051-1082, 2000.

[KLLV02] M. Kallahalla, K. Lee, B. Lee, and P. Varman. Performance comparison of sequential prefetch and forecasting in parallel I/O systems. Parallel and Distributed Systems and Networks, pages 76-84, 2002.

[KV] M. Kallahalla and P. Varman. Optimal read-once parallel disk scheduling: Online and offline algorithms. Algorithmica (to appear).

[KV99a] M. Kallahalla and P. J. Varman. Optimal read-once parallel disk scheduling. In Proc. of Sixth ACM Wkshp. on I/O in Parallel and Distributed Systems, pages 68-77, Atlanta, GA, 1999. ACM Press. 
[KV99b] M. Kallahalla and P. J. Varman. Randomized Parallel Prefetching and Buffer Management. In P. M. Pardalos and S. Rajasekaran, editors, Advances in Randomized Parallel Computing, chapter 9, pages 183-208. Kluwer Academic Publishers, 1999.

[KV01] M. Kallahalla and P. J. Varman. Optimal prefetching and caching for parallel I/O systems. In Proc. of 13th ACM Symp. on Parallel Algorithms and Architectures, 2001.

[KV02] M. Kallahalla and P. J. Varman. PC-OPT: optimal offline prefetching and caching for parallel I/O systems. IEEE Transactions on Computers, 51(11):1333-1344, Nov 2002.

[LS86] H. Luss and D. R. Smith. Resource allocation among competing activities. Operations Research Letters, 5(5):227-231, November 1986.

[McK99] Nick McKeown. The iSLIP scheduling algorithm for input-queued switches. IEEE/ACM Transactions On Networking, 7:188-201, April 1999.

[NB02] Nan Ni and Laxmi Narayan Bhuyan. Fair scheduling in internet routers. IEEE Transactions On Computers, pages 686-701, June 2002.

[NBBB98] K. Nichols, S. Blake, F. Baker, and D. Black. An architecture for differentiated services. IETF RFC 2475, December 1998.

[PGG $\left.{ }^{+} 95\right]$ R. H. Patterson, G. A. Gibson, E. Ginting, D. Stodolsky, and J. Zelenka. Informed prefetching and caching. In Proceedings of the fifteenth ACM Symposium on Operating Systems Principles, pages 79-95. ACM Press, 1995.

[PSV94] V. S. Pai, A. A. Schäffer, and P. J. Varman. Markov analysis of multiple-disk prefetching strategies for external merging. Theoretical Computer Science, 128(1-2):211-239, June 1994. 
[RVV04] Shah R., P. Varman, and J. S. Vitter. Online algorithms for prefetching and caching on parallel disks. Proc. 16th ACM Symposium on Parallelism in Algorithms and Architectures, (SPAA'04), June 2004.

[SEK00] P. Sanders, S. Egner, and J. H. M. Korst. Fast concurrent access to parallel disks. In Proc. of 11th ACM-SIAM Symp. on Discrete Algorithms, pages 849858 , January 2000.

[SOST93] T. E. Anderson S., S. Owicki, J. B. Saxe, and C. P. Thacker. High speed switch scheduling for local area networks. ACM Transactions On Computer Systems, 11:319-352, November 1993.

[SV98] Prashant J. Shenoy and Harrick M. Vin. Cello: a disk scheduling framework for next generation operating systems. In Proceedings of the 1998 ACM SIGMETRICS Joint International Conference On Measurement and Modeling of Computer Systems, pages 44-55. ACM Press, 1998.

[TS02] Leandros Tassiulas and Sawati Sarkar. Maxmin fair scheduling in wireless networks. IEEE INFOCOM, 2002.

[VS94] J. S. Vitter and E. A. M. Shriver. Optimal algorithms for parallel memory, I: Two-level memories. Algorithmica, 12(2-3):110-147, 1994.

[VV99] P. J. Varman and R. M. Verma. Tight bounds for prefetching and buffer management algorithms for parallel I/O systems. In IEEE Trans. on Parallel and Distributed Systems, volume 10, pages 1262-1275, December 1999.

[Wi101] John Wilkes. Traveling to Rome: QoS specifications for automated storage system management. In International Workshop on QoS, pages 75-91, June 2001.

[WPA ${ }^{+}$03] G. A. S. Whittle, J.-F. Paris, A. Amer, D. D. E. Long, and R. Burns. Using multiple predictors to improve the accuracy of file access predictions. In 20th 
IEEE Conference on Mass Storage Systems and Technologies, April 2003.

[Zha90] L. Zhang. Virtualclock: A new traffic control algorithm for packet switching networks. In SIGCOMM 90, pages 19-29, September 1990.

[ZL96] L. Q. Zheng and Per-Åke Larson. Speeding up external mergesort. IEEE Trans. on Knowledge and Data Engineering, 8(2):322-332, April 1996. 